\title{
RICCI FLAT KÄHLER METRICS WITH EDGE SINGULARITIES
}

\author{
SIMON BRENDLE
}

\begin{abstract}
We construct Ricci flat Kähler metrics with cone singularities along a complex hypersurface. This construction is inspired in part by R. Mazzeo's program in the case of negative Einstein constant, and uses the linear theory developed recently by S. Donaldson.
\end{abstract}

\section{INTRODUCTION}

Let $\left(M, \omega_{0}\right)$ be a compact Kähler manifold of complex dimension $n$, and let $\Sigma$ be a smooth complex hypersurface in $M$. Moreover, let $\Lambda$ denote the holomorphic line bundle associated to $\Sigma$, and let $h$ be a bundle metric on $\Lambda$. We are interested in Kähler metrics on $M \backslash \Sigma$ which have edge singularities along $\Sigma$. To construct an example of such a metric, let us fix a smooth Kähler metric $\omega_{0}$ on $M$; a holomorphic section $s$ of $\Lambda$; and a real number $\beta \in\left(0, \frac{1}{2}\right)$. If $\lambda>0$ is sufficiently small, then the $(1,1)$-form

$$
\omega=\omega_{0}+\lambda \sqrt{-1} \partial \bar{\partial}\left(|s|_{h}^{2 \beta}\right)
$$

defines a Kähler metric on $M \backslash \Sigma$ which has edge singularities along $\Sigma$. The number $\beta$ has a geometric interpretation in terms of the cone angle.

Our main result is an existence result for Ricci flat Kähler metrics with edge singularities.

Theorem 1.1. Let $\left(M, \omega_{0}\right)$ be a compact Kähler manifold of complex dimension $n$. Moreover, let $\Sigma$ be a smooth complex hypersurface in $M$; let $\Lambda$ denote the holomorphic line bundle associated to $\Sigma$; and let $\beta \in\left(0, \frac{1}{2}\right)$. Moreover, we assume that

$$
c_{1}(M)=(1-\beta) c_{1}(\Lambda) \in H^{1,1}(M, \mathbb{R}) .
$$

Then there exists a Kähler metric $\hat{\omega}=\omega+\sqrt{-1} \partial \bar{\partial} u$ on $M \backslash \Sigma$ with the following properties:

- The metric $\hat{\omega}$ is Ricci flat with bounded curvature.

- The metric $\hat{\omega}$ is uniformly equivalent to the background metric $\omega$, i.e. $a_{1} \omega \leq \hat{\omega} \leq a_{2} \omega$ for uniform constants $a_{1}, a_{2}>0$.

R. Mazzeo [8] has proposed a program for constructing Kähler-Einstein metrics with edge singularities in the case of negative Einstein constant. In dimension 2, this problem has been studied by M. Troyanov [13. Mazzeo's approach relies on the edge calculus developed in [7]. The edge calculus has 
proved to be a very useful tool in the study of various geometric problems. We also note that G. Tian and S.T Yau have constructed complete Ricci flat Kähler metrics on the complement of a divisor; see [11, [12 for details.

We now sketch the main steps involved in the proof of Theorem 1.1. In Section 2, we review some key results established in Donaldson's paper [3]. Of particular importance is the Schauder estimate proved in Donaldson's paper. This estimate plays a key role in the argument.

In Section 3, we describe the construction of the background metric $\omega$, and describe its basic properties. In particular, we show that $\operatorname{Ric}_{\omega}=\sqrt{-1} \partial \bar{\partial} F$ for some Hölder continuous function $F$. Moreover, we prove that $\omega$ has bounded curvature. Finally, we show that the covariant derivative of the curvature tensor of $\omega$ is bounded by $|D R|_{g} \leq C|\zeta|^{\varepsilon-\beta}$ for some $\varepsilon>0$.

It is well known that the metric $\hat{\omega}=\omega+\sqrt{-1} \partial \bar{\partial} u$ is Ricci flat if and only if the function $u$ satisfies the complex Monge-Ampère equation

$$
(\omega+\sqrt{-1} \partial \bar{\partial} u)^{n}=e^{F-c} \omega^{n}
$$

for some constant $c$. Following Aubin [1] and Yau [15], we solve this equation using the continuity method. To that end, we consider the equation

$$
(\omega+\sqrt{-1} \partial \bar{\partial} u)^{n}=e^{t F-c} \omega^{n},
$$

where $t \in[0,1]$ is a parameter.

In Section 4, we establish a uniform $C^{0}$-estimate for solutions of $\left(\star_{t}\right)$. More precisely, we show that $\sup _{M} u-\inf _{M} u \leq C$ for any solution $u \in \mathcal{C}^{2, \alpha, \beta}$ of $\left(\star_{t}\right)$.

In Section 5, we establish a Laplacian estimate for solutions of $\left(\star_{t}\right)$. In particular, this estimate implies that $a_{1} \omega \leq \hat{\omega} \leq a_{2} \omega$ whenever $u \in \mathcal{C}^{2, \alpha, \beta}$ is a solution of $\left(\star_{t}\right)$. In order to prove the Laplacian estimate, we adapt the arguments used in Yau's proof of the Calabi conjecture (cf. [14, [15]). Our situation is more subtle in that we are dealing with a singular background metric $\omega$. Furthermore, the application of the maximum principle is not entirely straightforward, as the maximum may be attained on the singular set. In order to make the maximum principle work, we use a trick due to T. Jeffres [5], 6].

In Section 6, we obtain an a-priori estimate for the covariant derivative of $\partial \bar{\partial} u$. This estimate is again proved using the maximum principle. In order to apply the maximum principle, we need to analyze the asymptotic behavior of the third order covariant derivatives of $u$ near $\Sigma$.

Finally, in Section [7, we show that the equation $\left(\star_{1}\right)$ has a solution $u \in$ $\mathcal{C}^{2, \alpha, \beta}$. Moreover, we show that the associated Kähler metric $\hat{\omega}=\omega+$

$\sqrt{-1} \partial \bar{\partial} u$ has bounded curvature, thereby completing the proof of Theorem 1.1 .

\section{DonAldSON'S WORK ON THE LINEAR THEORY}

In this section, we collect some results proved in Donaldson's paper 3, which play a major role in our argument. These results are all due to 
Donaldson, and are described here for the convenience of the reader. To begin with, we consider the Kähler metric

$$
\begin{aligned}
\Omega & =\sqrt{-1} \partial \bar{\partial}\left(\left|z_{1}\right|^{2}+\ldots+\left|z_{n-1}\right|^{2}+|\zeta|^{2 \beta}\right) \\
& =\sum_{k=1}^{n-1} \sqrt{-1} d z_{k} \wedge d \bar{z}_{k}+\beta^{2} \sqrt{-1}|\zeta|^{2 \beta-2} d \zeta \wedge d \bar{\zeta}
\end{aligned}
$$

on $\mathbb{C}^{n}$. The Laplacian with respect to that metric is given by

$$
\Delta_{\Omega} v=\sum_{k=1}^{n} \frac{\partial^{2} v}{\partial z_{k} \partial \bar{z}_{k}}+\frac{1}{\beta^{2}}|\zeta|^{2-2 \beta} \frac{\partial^{2} v}{\partial \zeta \partial \bar{\zeta}} .
$$

The goal is to solve the equation $\Delta_{\Omega} v=f$. To that end, we write

$$
v\left(z_{1}, \ldots, z_{n-1}, \zeta\right)=\tilde{v}\left(z_{1}, \ldots, z_{n-1},|\zeta|^{\beta-1} \zeta\right)
$$

and

$$
f\left(z_{1}, \ldots, z_{n-1}, \zeta\right)=\tilde{f}\left(z_{1}, \ldots, z_{n-1},|\zeta|^{\beta-1} \zeta\right) .
$$

The equation $\Delta_{\Omega} v=f$ is then equivalent to the equation

$$
\sum_{k=1}^{n-1} \frac{\partial^{2} \tilde{v}}{\partial z_{k} \partial \bar{z}_{k}}+\frac{1}{4}\left(\frac{\partial^{2} \tilde{v}}{\partial r^{2}}+\frac{1}{r} \frac{\partial \tilde{v}}{\partial r}+\frac{1}{\beta^{2} r^{2}} \frac{\partial^{2} \tilde{v}}{\partial \theta^{2}}\right)=\tilde{f} .
$$

Here, $|\zeta|^{\beta-1} \zeta=\xi=r e^{i \theta}$.

Proposition 2.1 (S. Donaldson [3]). Let $\tilde{f}$ be a function of class $C^{\alpha}$ with compact support. Then the equation (11) has a unique weak solution $\tilde{v} \in$ $L^{\frac{2 n}{n-1}}\left(\mathbb{C}^{n}\right)$. The function $\tilde{v}$ is of class $C^{1, \alpha}$ and satisfies

$$
\frac{\partial \tilde{v}}{\partial \xi}\left(z_{1}, \ldots, z_{n-1}, 0\right)=\frac{\partial \tilde{v}}{\partial \bar{\xi}}\left(z_{1}, \ldots, z_{n-1}, 0\right)=0 .
$$

Moreover, we have the estimate

$$
\begin{gathered}
\sum_{1 \leq k, l \leq n-1}\left[\frac{\partial^{2} \tilde{v}}{\partial z_{k} \partial z_{l}}\right]_{C^{\alpha}}+\sum_{1 \leq k, l \leq n-1}\left[\frac{\partial^{2} \tilde{v}}{\partial z_{k} \partial \bar{z}_{l}}\right]_{C^{\alpha}} \\
+\sum_{k=1}^{n-1}\left[\frac{\partial^{2} \tilde{v}}{\partial z_{k} \partial \xi}\right]_{C^{\alpha}}+\sum_{k=1}^{n-1}\left[\frac{\partial^{2} \tilde{v}}{\partial z_{k} \partial \bar{\xi}}\right]_{C^{\alpha}} \leq C[\tilde{f}]_{C^{\alpha}} .
\end{gathered}
$$

The identity (21) follows from the polyhomogeneous expansion for the Green's function derived in Donaldson's paper. By differentiating identity (2) in tangential direction, Donaldson showed that

$$
\frac{\partial^{2} \tilde{v}}{\partial z_{k} \partial \xi}\left(z_{1}, \ldots, z_{n-1}, 0\right)=\frac{\partial^{2} \tilde{v}}{\partial z_{k} \partial \bar{\xi}}\left(z_{1}, \ldots, z_{n-1}, 0\right)=0
$$

for each $k \in\{1, \ldots, n-1\}$.

Let us recall some notation from Donaldson's paper [3]. Let $\mathcal{H}$ be the space of all functions $f$ of the form $f\left(z_{1}, \ldots, z_{n-1}, \zeta\right)=\tilde{f}\left(z_{1}, \ldots, z_{n-1},|\zeta|^{\beta-1} \zeta\right)$, where $\tilde{f} \in W^{1,2}$. As in [3], we denote by $\mathcal{C}^{, \alpha, \beta}$ the space of all functions $f$ of 
the form $f\left(z_{1}, \ldots, z_{n-1}, \zeta\right)=\tilde{f}\left(z_{1}, \ldots, z_{n-1},|\zeta|^{\beta-1} \zeta\right)$ where $\tilde{f} \in C^{\alpha}$. Moreover, let us denote by $\mathcal{C}_{0}^{, \alpha, \beta}$ the space of all functions $f \in \mathcal{C}^{, \alpha, \beta}$ such that $f\left(z_{1}, \ldots, z_{n-1}, 0\right)=0$. A $(1,0)$-form $\tau$ is said to be of class $\mathcal{C}^{, \alpha, \beta}$ if

$$
\begin{aligned}
& \tau\left(\frac{\partial}{\partial z_{k}}\right) \in \mathcal{C}^{, \alpha, \beta}, \\
& |\zeta|^{1-\beta} \tau\left(\frac{\partial}{\partial \zeta}\right) \in \mathcal{C}_{0}^{, \alpha, \beta} .
\end{aligned}
$$

Moreover, a $(1,1)$-form $\sigma$ is said to be of class $\mathcal{C}^{, \alpha, \beta}$ if

$$
\begin{aligned}
& \sigma\left(\frac{\partial}{\partial z_{k}}, \frac{\partial}{\partial \bar{z}_{l}}\right) \in \mathcal{C}^{, \alpha, \beta}, \\
& |\zeta|^{1-\beta} \sigma\left(\frac{\partial}{\partial z_{k}}, \frac{\partial}{\partial \bar{\zeta}}\right) \in \mathcal{C}_{0}^{, \alpha, \beta} \\
& |\zeta|^{1-\beta} \sigma\left(\frac{\partial}{\partial \zeta}, \frac{\partial}{\partial \bar{z}_{l}}\right) \in \mathcal{C}_{0}^{, \alpha, \beta} \\
& |\zeta|^{2-2 \beta} \sigma\left(\frac{\partial}{\partial \zeta}, \frac{\partial}{\partial \bar{\zeta}}\right) \in \mathcal{C}^{, \alpha, \beta}
\end{aligned}
$$

Finally, let

$$
\mathcal{C}^{2, \alpha, \beta}=\left\{v \in C^{2}(M \backslash \Sigma): v, \partial v, \partial \bar{\partial} v \text { are of class } \mathcal{C}^{, \alpha, \beta}\right\}
$$

(cf. [3, p. 16).

Consider now a function $f \in \mathcal{C}^{, \alpha, \beta}$ which is supported in the ball $B_{6}(0)$. In view of Donaldson's results, the equation $\Delta_{\Omega} v=f$ admits a weak solution $v \in \mathcal{H}$ satisfying $\int_{\mathbb{C}^{n}}|v|^{\frac{2 n}{n-1}} \Omega^{n}<\infty$. The Schauder estimate (3) implies

$$
\sum_{1 \leq k, l \leq n-1}\left\|\frac{\partial^{2} v}{\partial z_{k} \partial z_{l}}\right\|_{\mathcal{C}, \alpha, \beta}+\sum_{1 \leq k, l \leq n-1}\left\|\frac{\partial^{2} v}{\partial z_{k} \partial \bar{z}_{l}}\right\|_{\mathcal{C}, \alpha, \beta} \leq C\|f\|_{\mathcal{C}, \alpha, \beta} .
$$

Using the equation $\Delta_{\Omega} v=f$, Donaldson obtained the estimate

$$
\left\||\zeta|^{2 \beta-2} \frac{\partial^{2} v}{\partial \zeta \partial \bar{\zeta}}\right\|_{\mathcal{C}, \alpha, \beta} \leq C\|f\|_{\mathcal{C}, \alpha, \beta} .
$$

We next observe that

$$
\begin{aligned}
|\zeta|^{1-\beta} \frac{\partial^{2} v}{\partial z_{k} \partial \bar{\zeta}}\left(z_{1}, \ldots, z_{n-1}, \zeta\right) & =\frac{\beta-1}{2} \frac{\zeta^{2}}{|\zeta|^{2}} \frac{\partial^{2} \tilde{v}}{\partial z_{k} \partial \xi}\left(z_{1}, \ldots, z_{n-1},|\zeta|^{\beta-1} \zeta\right) \\
& +\frac{\beta+1}{2} \frac{\partial^{2} \tilde{v}}{\partial z_{k} \partial \bar{\xi}}\left(z_{1}, \ldots, z_{n-1},|\zeta|^{\beta-1} \zeta\right)
\end{aligned}
$$

for $k \in\{1, \ldots, n-1\}$. In view of (4), the functions

$$
\left(z_{1}, \ldots, z_{n-1}, \zeta\right) \mapsto \frac{\partial^{2} \tilde{v}}{\partial z_{k} \partial \xi}\left(z_{1}, \ldots, z_{n-1},|\zeta|^{\beta-1} \zeta\right)
$$

and

$$
\left(z_{1}, \ldots, z_{n-1}, \zeta\right) \mapsto \frac{\partial^{2} \tilde{v}}{\partial z_{k} \partial \bar{\xi}}\left(z_{1}, \ldots, z_{n-1},|\zeta|^{\beta-1} \zeta\right)
$$


belong to the space $\mathcal{C}_{0}^{, \alpha, \beta}$. Consequently, the function $|\zeta|^{1-\beta} \frac{\partial^{2} v}{\partial z_{k} \partial \zeta}$ belongs to the space $\mathcal{C}_{0}^{, \alpha, \beta}$, and one has the estimate

$$
\left\||\zeta|^{1-\beta} \frac{\partial^{2} v}{\partial z_{k} \partial \bar{\zeta}}\right\|_{\mathcal{C}, \alpha, \beta} \leq C\|f\|_{\mathcal{C}, \alpha, \beta} .
$$

Donaldson's Schauder estimate can be summarized as follows:

Proposition 2.2 (S. Donaldson [3]). Let $f \in \mathcal{C}^{, \alpha, \beta}$ be a function supported in the ball $B_{6}(0)$. Then there exists a unique weak solution $v \in \mathcal{H}$ of the equation $\Delta_{\Omega} v=f$ such that $\int_{\mathbb{C}^{n}}|v|^{\frac{2 n}{n-1}} \Omega^{n}<\infty$. Moreover, $v$ is of class $\mathcal{C}^{2, \alpha, \beta}$ and we have the estimate

$$
\|v\|_{\mathcal{C}^{2, \alpha, \beta}} \leq C\|f\|_{\mathcal{C}, \alpha, \beta} .
$$

It is shown in Section 4.2 of Donaldson's paper that the Schauder estimate in Proposition 2.2 carries over to the variable coefficient setting. In the following, $\omega$ will denote a Kähler metric on the ball $B_{6}(0)$ with the property that $\omega$ is of class $\mathcal{C}^{, \alpha, \beta}$ and $\|\omega-\Omega\|_{\mathcal{C}, \alpha, \beta} \leq \varepsilon_{0}$. Here, $\varepsilon_{0}$ denotes a small positive constant.

Proposition 2.3 (S. Donaldson [3]). Let $\omega$ be a Kähler metric on the ball $B_{6}(0)$ such that $\omega$ is of class $\mathcal{C}^{, \alpha, \beta}$ and $\|\omega-\Omega\|_{\mathcal{C}, \alpha, \beta} \leq \varepsilon_{0}$. Moreover, let $v$ be a function of class $\mathcal{C}^{2, \alpha, \beta}$ defined on the ball $B_{2}(0)$. If $\varepsilon_{0}$ is sufficiently small, then we have the estimate

$$
\|v\|_{\mathcal{C}^{2, \alpha, \beta}\left(B_{1}(0)\right)} \leq C \sup _{B_{2}(0)}|v|+C\left\|\Delta_{\omega} v\right\|_{\mathcal{C}, \alpha, \beta}\left(B_{2}(0)\right) .
$$

Proposition 2.3 is a consequence of Proposition 2.2 (compare [9]). Note that Proposition 2.3 presupposes that the function $v$ is of class $\mathcal{C}^{2, \alpha, \beta}$. This assumption can be removed as follows:

Proposition 2.4 (S. Donaldson 3]). Let $\omega$ be a Kähler metric on the ball $B_{6}(0)$ which is of class $\mathcal{C}, \alpha, \beta$ and satisfies $\|\omega-\Omega\|_{\mathcal{C}, \alpha, \beta} \leq \varepsilon_{0}$. Suppose that $\varphi$ is a function of class $\mathcal{C}^{, \alpha, \beta}$ defined on the ball $B_{6}(0)$, and $v \in \mathcal{H}$ is a weak solution of the equation $\Delta_{\omega} v=\varphi$. If $\varepsilon_{0}$ is sufficiently small, then the restriction $\left.v\right|_{B_{1}(0)}$ is of class $\mathcal{C}^{2, \alpha, \beta}$.

We define

$$
\mathcal{X}=\left\{f \in \mathcal{C}^{, \alpha, \beta}: f \text { vanishes on } \mathbb{C}^{n} \backslash B_{6}(0)\right\} .
$$

Given any function $f \in \mathcal{X}$, we denote by $v=G f$ the unique weak solution of the equation $\Delta_{\Omega} v=f$. By Proposition 2.2, this defines a bounded linear operator $G: \mathcal{X} \rightarrow \mathcal{C}^{2, \alpha, \beta}$.

Let us fix a smooth cutoff function $\chi$ such that $\chi=1$ in $B_{5}(0)$ and $\chi=0$ on $\mathbb{C}^{n} \backslash B_{6}(0)$. We define a differential operator $L$ by

$$
L v=\sqrt{-1} \frac{\partial((1-\chi) \bar{\partial} v) \wedge \Omega^{n-1}+\partial(\chi \bar{\partial} v) \wedge \omega^{n-1}}{(1-\chi) \Omega^{n}+\chi \omega^{n}} .
$$


Clearly, $L v=\Delta_{\Omega} v$ on $\mathbb{C}^{n} \backslash B_{6}(0)$. Consequently, the operator $L G: \mathcal{X} \rightarrow$ $\mathcal{C}^{, \alpha, \beta}$ maps $\mathcal{X}$ into itself. Moreover, we have $\|L G f-f\|_{\mathcal{C}, \alpha, \beta} \leq C \varepsilon_{0}\|f\|_{\mathcal{C}, \alpha, \beta}$. Hence, if $\varepsilon_{0}$ is small enough, then the operator $L G: \mathcal{X} \rightarrow \mathcal{X}$ is invertible. Let $T: \mathcal{X} \rightarrow \mathcal{X}$ denote the inverse of $L G$.

We now sketch the proof of Proposition 2.4. Let $\eta$ be a smooth cutoff function satisfying $\eta=1$ in $B_{4}(0)$ and $\eta=0$ in $\mathbb{C}^{n} \backslash B_{5}(0)$, and let $\psi=$ $\eta \varphi+2\langle d \eta, d v\rangle_{\omega}+v \Delta_{\omega} \eta$. Then $\int_{B_{6}(0)}|\psi|^{2} \Omega^{n}<\infty$ and the restriction $\left.\psi\right|_{B_{4}(0)}$ is of class $\mathcal{C}^{, \alpha, \beta}$. We can find a sequence of functions $\psi_{k} \in \mathcal{X}$ such that $\left.\psi_{k}\right|_{B_{3}(0)}=\left.\psi\right|_{B_{3}(0)}$ and

$$
\int_{B_{6}(0)}\left|\psi-\psi_{k}\right|^{2} \Omega^{n} \rightarrow 0
$$

as $k \rightarrow \infty$. For each $k$, the function $v_{k}=G T \psi_{k}$ is of class $\mathcal{C}^{2, \alpha, \beta}$, and we have $L v_{k}=\psi_{k}$ away from the set $\{\zeta=0\}$. On the other hand, the function $\eta v \in \mathcal{H}$ is a weak solution of the equation $L(\eta v)=\psi$. Consequently, the function $\eta v-v_{k} \in \mathcal{H}$ is a weak solution of the equation $L\left(\eta v-v_{k}\right)=\psi-\psi_{k}$. Using (5), we obtain

$$
\int_{\mathbb{C}^{n}}\left|\eta v-v_{k}\right|^{\frac{2 n}{n-1}} \Omega^{n} \rightarrow 0
$$

as $k \rightarrow \infty$.

Note that the pull-back of the Kähler metric $\omega$ under the map $\Phi$ : $\left(z_{1}, \ldots, z_{n-1}, \xi\right) \mapsto\left(z_{1}, \ldots, z_{n-1},|\xi|^{\frac{1}{\beta}-1} \xi\right)$ is uniformly equivalent to the Euclidean metric. Hence, it follows from Theorem 8.17 in [4] that

$$
\sup _{B_{2}(0)}\left|v_{k}\right| \leq C\left(\int_{B_{3}(0)}\left|v_{k}\right|^{\frac{2 n}{n-1}} \Omega^{n}\right)^{\frac{n-1}{2 n}}+C \sup _{B_{3}(0)}\left|\Delta_{\omega} v_{k}\right| .
$$

Moreover, since $v_{k} \in \mathcal{C}^{2, \alpha, \beta}$, we have

$$
\left\|v_{k}\right\|_{\mathcal{C}^{2, \alpha, \beta}\left(B_{1}(0)\right)} \leq C \sup _{B_{2}(0)}\left|v_{k}\right|+C\left\|\Delta_{\omega} v_{k}\right\|_{\mathcal{C}, \alpha, \beta}\left(B_{2}(0)\right)
$$

by Proposition 2.3. On the other hand, we have $\Delta_{\omega} v_{k}=L v_{k}=\psi_{k}=\psi$ in $B_{3}(0)$. Putting these facts together, we obtain

$$
\left\|v_{k}\right\|_{\mathcal{C}^{2, \alpha, \beta}\left(B_{1}(0)\right)} \leq C\left(\int_{B_{3}(0)}\left|v_{k}\right|^{\frac{2 n}{n-1}} \Omega^{n}\right)^{\frac{n-1}{2 n}}+C\|\psi\|_{\mathcal{C}, \alpha, \beta}\left(B_{3}(0)\right)
$$

for some uniform constant $C$. Using (6) , we conclude that $\left.v\right|_{B_{1}(0)} \in \mathcal{C}^{2, \alpha, \beta}$, as claimed.

By freezing coefficients, Donaldson obtained the following regularity result:

Theorem 2.5 (S. Donaldson [3]). Let $\omega$ be a Kähler metric on the ball $B_{6}(0)$ which is of class $\mathcal{C}^{, \alpha, \beta}$ and satisfies $a_{1} \Omega \leq \omega \leq a_{2} \Omega$ for suitable constants $a_{1}, a_{2}>0$. Suppose that $\varphi$ is a function of class $\mathcal{C}^{, \alpha, \beta}$ defined on the ball 
$B_{6}(0)$, and $v \in \mathcal{H}$ is a weak solution of the equation $\Delta_{\omega} v=\varphi$. Then the restriction $\left.v\right|_{B_{1}(0)}$ is of class $\mathcal{C}^{2, \alpha, \beta}$.

The following is a special case of Theorem 2.5.

Corollary 2.6 (S. Donaldson [3]). Let $\omega$ be a Kähler metric on the ball $B_{6}(0)$ which is of class $\mathcal{C}^{, \alpha, \beta}$ and satisfies $a_{1} \Omega \leq \omega \leq a_{2} \Omega$ for suitable constants $a_{1}, a_{2}>0$. Suppose that $v$ and $\varphi$ are functions of class $\mathcal{C}^{, \alpha, \beta}$ defined on the ball $B_{6}(0)$ such that $\Delta_{\omega} v=\varphi$ away from the set $\{\zeta=0\}$. Then the restriction $\left.v\right|_{B_{1}(0)}$ is of class $\mathcal{C}^{2, \alpha, \beta}$.

\section{The BACKGRound EDGE MetriC}

In the following, we fix a Kähler manifold $\left(M, \omega_{0}\right)$, a smooth complex hypersurface $\Sigma \subset M$, and a real number $\beta \in\left(0, \frac{1}{2}\right)$. Let $\Lambda$ be the holomorphic line bundle associated with $\Sigma$, and let $s$ be a holomorphic section of $\Lambda$ such that $\Sigma=\{s=0\}$.

The $(1,1)$-form $-\sqrt{-1} \partial \bar{\partial} \log \left(|s|_{h}^{2}\right)$ is smooth and represents the cohomology class $c_{1}(\Lambda) \in H^{1,1}(M, \mathbb{R})$. Consequently, the cohomology class $c_{1}(M)-(1-\beta) c_{1}(\Lambda) \in H^{1,1}(M, \mathbb{R})$ can be represented by the $(1,1)$-form

$$
\operatorname{Ric}_{\omega_{0}}+(1-\beta) \sqrt{-1} \partial \bar{\partial} \log \left(|s|_{h}^{2}\right) .
$$

Since $c_{1}(M)=(1-\beta) c_{1}(\Lambda)$, there exists a smooth function $F_{0}$ such that

$$
\operatorname{Ric}_{\omega_{0}}+(1-\beta) \sqrt{-1} \partial \bar{\partial} \log \left(|s|_{h}^{2}\right)=\sqrt{-1} \partial \bar{\partial} F_{0} .
$$

We next define

$$
\omega=\omega_{0}+\lambda \sqrt{-1} \partial \bar{\partial}\left(|s|_{h}^{2 \beta}\right)
$$

It is easy to see that

$$
\omega \geq \omega_{0}+\lambda \beta|s|_{h}^{2 \beta} \sqrt{-1} \partial \bar{\partial} \log \left(|s|_{h}^{2}\right) .
$$

The term $\partial \bar{\partial} \log \left(|s|_{h}^{2}\right)$ defines a smooth $(1,1)$-form on $M$. Hence, if we choose $\lambda>0$ sufficiently small, then $\omega$ is a Kähler metric on $M \backslash \Sigma$. The Ricci curvature of $\omega$ is given by

$$
\begin{aligned}
\operatorname{Ric}_{\omega} & =\operatorname{Ric}_{\omega_{0}}-\sqrt{-1} \partial \bar{\partial} \log \frac{\omega^{n}}{\omega_{0}^{n}} \\
& =\sqrt{-1} \partial \bar{\partial} F_{0}-(1-\beta) \sqrt{-1} \partial \bar{\partial} \log \left(|s|_{h}^{2}\right)-\sqrt{-1} \partial \bar{\partial} \log \frac{\omega^{n}}{\omega_{0}^{n}} \\
& =\sqrt{-1} \partial \bar{\partial} F
\end{aligned}
$$

where

$$
F=F_{0}-\log \frac{|s|_{h}^{2-2 \beta} \omega^{n}}{\omega_{0}^{n}} .
$$

Clearly, $F$ is a smooth function away from $\Sigma$. We next analyze the behavior of $F$ near $\Sigma$. To that end, we fix a point $p \in \Sigma$ and introduce complex 
coordinates $\left(z_{1}, \ldots, z_{n-1}, \zeta\right)$ near $p$ so that $\Sigma=\{\zeta=0\}$. Moreover, we write $|s|_{h}^{2 \beta}=\rho|\zeta|^{2 \beta}$, where $\rho$ is a smooth positive function. Then

$$
\omega=\omega_{0}+\lambda \sqrt{-1} \partial \bar{\partial}\left(\rho|\zeta|^{2 \beta}\right)
$$

hence

$$
\begin{aligned}
\omega & =\omega_{0}+\lambda|\zeta|^{2 \beta} \sqrt{-1} \partial \bar{\partial} \rho \\
& +\lambda \beta|\zeta|^{2 \beta-2} \zeta \sqrt{-1} \partial \rho \wedge d \bar{\zeta} \\
& +\lambda \beta|\zeta|^{2 \beta-2} \bar{\zeta} \sqrt{-1} d \zeta \wedge \bar{\partial} \rho \\
& +\lambda \beta^{2} \rho \sqrt{-1}|\zeta|^{2 \beta-2} d \zeta \wedge d \bar{\zeta}
\end{aligned}
$$

This implies

$$
\frac{\omega^{n}}{\omega_{0}^{n}}=|\zeta|^{2 \beta-2} \sum_{m=0}^{n-1} a_{m}|\zeta|^{2 \beta m}+\sum_{m=0}^{n} b_{m}|\zeta|^{2 \beta m},
$$

where $a_{m}, b_{m}$ are smooth functions and $a_{0}$ is positive. Consequently,

$$
\begin{aligned}
\frac{|s|_{h}^{2-2 \beta} \omega^{n}}{\omega_{0}^{n}} & =\rho^{\frac{1}{\beta}-1} \frac{|\zeta|^{2-2 \beta} \omega^{n}}{\omega_{0}^{n}} \\
& =\rho^{\frac{1}{\beta}-1}\left(\sum_{m=0}^{n-1} a_{m}|\zeta|^{2 \beta m}+|\zeta|^{2-2 \beta} \sum_{m=0}^{n} b_{m}|\zeta|^{2 \beta m}\right),
\end{aligned}
$$

hence

$$
\begin{aligned}
F & =F_{0}-\left(\frac{1}{\beta}-1\right) \log \rho \\
& -\log \left(\sum_{m=0}^{n-1} a_{m}|\zeta|^{2 \beta m}+|\zeta|^{2-2 \beta} \sum_{m=0}^{n} b_{m}|\zeta|^{2 \beta m}\right) .
\end{aligned}
$$

Since $a_{m}, b_{m}$, and $\rho$ are smooth functions, we can draw the following conclusions:

Proposition 3.1. The function F is Hölder continuous. Moreover, we have

$$
|\partial \bar{\partial} F|_{g} \leq C+C|\zeta|^{2-4 \beta} \text {. }
$$

In particular, if $\beta \in\left(0, \frac{1}{2}\right)$, then $|\partial \bar{\partial} F|_{g} \leq C$.

In the next step, we estimate the Riemann curvature tensor of $g$. As above, we may work in local complex coordinates around a point $p \in \Sigma$. We will consider the pull-back of $\omega$ under the holomorphic map

$$
\Psi:\left(z_{1}, \ldots, z_{n-1}, z_{n}\right) \mapsto\left(z_{1}, \ldots, z_{n-1}, z_{n}^{\frac{1}{\beta}}\right) .
$$

Note that $\Psi$ is defined only locally. Using the identity (7), we obtain

$$
\Psi^{*} \omega=\Psi^{*} \omega_{0}+\lambda \sqrt{-1} \partial \bar{\partial}\left((\rho \circ \Psi)\left|z_{n}\right|^{2}\right),
$$


hence

$$
\begin{aligned}
\Psi^{*} \omega & =\Psi^{*} \omega_{0}+\lambda\left|z_{n}\right|^{2} \sqrt{-1} \partial \bar{\partial}(\rho \circ \Psi) \\
& +\lambda z_{n} \sqrt{-1} \partial(\rho \circ \Psi) \wedge d \bar{z}_{n} \\
& +\lambda \bar{z}_{n} \sqrt{-1} d z_{n} \wedge \bar{\partial}(\rho \circ \Psi) \\
& +\lambda(\rho \circ \Psi) \sqrt{-1} d z_{n} \wedge d \bar{z}_{n} .
\end{aligned}
$$

This implies

$$
\Psi^{*} g\left(\frac{\partial}{\partial z_{k}}, \frac{\partial}{\partial \bar{z}_{l}}\right)=\left(g_{0, k \bar{l}} \circ \Psi\right)+\lambda\left|z_{n}\right|^{2}\left(\frac{\partial^{2} \rho}{\partial z_{k} \partial \bar{z}_{l}} \circ \Psi\right)
$$

and

$$
\begin{aligned}
\Psi^{*} g\left(\frac{\partial}{\partial z_{n}}, \frac{\partial}{\partial \bar{z}_{l}}\right) & =\frac{1}{\beta} z_{n}^{\frac{1}{\beta}-1}\left(g_{0, \zeta \bar{l}} \circ \Psi\right)+\frac{\lambda}{\beta} z_{n}^{\frac{1}{\beta}} \bar{z}_{n}\left(\frac{\partial^{2} \rho}{\partial \zeta \partial \bar{z}_{l}} \circ \Psi\right) \\
& +\lambda \bar{z}_{n}\left(\frac{\partial \rho}{\partial \bar{z}_{l}} \circ \Psi\right)
\end{aligned}
$$

for all $k, l \in\{1, \ldots, n-1\}$. Moreover, we have

$$
\begin{aligned}
\Psi^{*} g\left(\frac{\partial}{\partial z_{n}}, \frac{\partial}{\partial \bar{z}_{n}}\right) & =\frac{1}{\beta^{2}}\left|z_{n}\right|^{\frac{2}{\beta}-2}\left(g_{0, \zeta \bar{\zeta}} \circ \Psi\right)+\frac{\lambda}{\beta^{2}}\left|z_{n}\right|^{\frac{2}{\beta}}\left(\frac{\partial^{2} \rho}{\partial \zeta \partial \bar{\zeta}} \circ \Psi\right) \\
& +\frac{\lambda}{\beta} z_{n}^{\frac{1}{\beta}}\left(\frac{\partial \rho}{\partial \zeta} \circ \Psi\right)+\frac{\lambda}{\beta} \bar{z}_{n}^{\frac{1}{\beta}}\left(\frac{\partial \rho}{\partial \bar{\zeta}^{\prime}} \circ \Psi\right)+\lambda(\rho \circ \Psi) .
\end{aligned}
$$

Using these identities, we obtain estimates for the first derivatives of $\Psi^{*} g$.

Lemma 3.2. We have

$$
\begin{aligned}
& \sum_{i, k, l=1}^{n-1}\left|\frac{\partial}{\partial z_{i}} \Psi^{*} g\left(\frac{\partial}{\partial z_{k}}, \frac{\partial}{\partial \bar{z}_{l}}\right)\right| \leq C, \\
& \sum_{i, l=1}^{n-1}\left|\frac{\partial}{\partial z_{i}} \Psi^{*} g\left(\frac{\partial}{\partial z_{n}}, \frac{\partial}{\partial \bar{z}_{l}}\right)\right| \leq C\left|z_{n}\right|+C\left|z_{n}\right|^{\frac{1}{\beta}-1}, \\
& \sum_{i=1}^{n-1}\left|\frac{\partial}{\partial z_{i}} \Psi^{*} g\left(\frac{\partial}{\partial z_{n}}, \frac{\partial}{\partial \bar{z}_{n}}\right)\right| \leq C, \\
& \sum_{l=1}^{n-1}\left|\frac{\partial}{\partial z_{n}} \Psi^{*} g\left(\frac{\partial}{\partial z_{n}}, \frac{\partial}{\partial \bar{z}_{l}}\right)\right| \leq C\left|z_{n}\right|^{\frac{1}{\beta}-2}, \\
& \left|\frac{\partial}{\partial z_{n}} \Psi^{*} g\left(\frac{\partial}{\partial z_{n}}, \frac{\partial}{\partial \bar{z}_{n}}\right)\right| \leq C\left|z_{n}\right|^{\frac{1}{\beta}-1}+C\left|z_{n}\right|^{\frac{2}{\beta}-3} .
\end{aligned}
$$

The second derivatives of $\Psi^{*} g$ can be estimated as follows: 
Lemma 3.3. We have

$$
\begin{aligned}
& \sum_{i, j, k, l=1}^{n-1}\left|\frac{\partial^{2}}{\partial z_{i} \partial \bar{z}_{j}} \Psi^{*} g\left(\frac{\partial}{\partial z_{k}}, \frac{\partial}{\partial \bar{z}_{l}}\right)\right| \leq C, \\
& \sum_{i, j, l=1}^{n-1}\left|\frac{\partial^{2}}{\partial z_{i} \partial \bar{z}_{j}} \Psi^{*} g\left(\frac{\partial}{\partial z_{n}}, \frac{\partial}{\partial \bar{z}_{l}}\right)\right| \leq C\left|z_{n}\right|+C\left|z_{n}\right|^{\frac{1}{\beta}-1}, \\
& \sum_{i, j=1}^{n}\left|\frac{\partial^{2}}{\partial z_{i} \partial \bar{z}_{j}} \Psi^{*} g\left(\frac{\partial}{\partial z_{n}}, \frac{\partial}{\partial \bar{z}_{n}}\right)\right| \leq C, \\
& \sum_{j, l=1}^{n}\left|\frac{\partial^{2}}{\partial z_{n} \partial \bar{z}_{j}} \Psi^{*} g\left(\frac{\partial}{\partial z_{n}}, \frac{\partial}{\partial \bar{z}_{l}}\right)\right| \leq C\left|z_{n}\right|^{\frac{1}{\beta}-2}, \\
& \sum_{i=1}^{n}\left|\frac{\partial^{2}}{\partial z_{i} \partial \bar{z}_{n}} \Psi^{*} g\left(\frac{\partial}{\partial z_{n}}, \frac{\partial}{\partial \bar{z}_{n}}\right)\right| \leq C\left|z_{n}\right|^{\frac{1}{\beta}-1}+C\left|z_{n}\right|^{\frac{2}{\beta}-3}, \\
& \left|\frac{\partial^{2}}{\partial z_{n} \partial \bar{z}_{n}} \Psi^{*} g\left(\frac{\partial}{\partial z_{n}}, \frac{\partial}{\partial \bar{z}_{n}}\right)\right| \leq C\left|z_{n}\right|^{\frac{2}{\beta}-4} .
\end{aligned}
$$

We also note that

$$
\left|\frac{\partial^{2}}{\partial z_{n} \partial z_{n}} \Psi^{*} g\left(\frac{\partial}{\partial z_{n}}, \frac{\partial}{\partial \bar{z}_{l}}\right)\right| \leq C\left|z_{n}\right|^{\frac{1}{\beta}-3} .
$$

Note that this term does not enter into the formula for the curvature tensor.

After these preparations, we now derive a bound for the Riemann curvature tensor of $g$.

Proposition 3.4. The curvature tensor of $g$ can be estimated by

$$
|R|_{g} \leq C+C\left|z_{n}\right|^{\frac{1}{\beta}-2}+C\left|z_{n}\right|^{\frac{2}{\beta}-4} \text {. }
$$

In particular, if $\beta \in\left(0, \frac{1}{2}\right)$, then the curvature tensor of $\omega$ is bounded.

Proof. The curvature tensor of a Kähler manifold is given by

$$
R_{\alpha \bar{\beta} \gamma \bar{\delta}}=-\partial_{\alpha} \partial_{\bar{\beta}} g_{\gamma \bar{\delta}}+\sum_{\mu, \nu=1}^{n} g^{\mu \bar{\nu}} \partial_{\gamma} g_{\alpha \bar{\nu}} \partial_{\bar{\delta}} g_{\mu \bar{\beta}}
$$

(cf. [15], page 344). Note that the metric $\Psi^{*} g$ is uniformly equivalent to the Euclidean metric. Hence, it follows from Lemma 3.2 and Lemma 3.3 that

$$
\begin{aligned}
|R|_{g} & \leq C \sum_{\alpha, \beta, \gamma, \delta}^{n}\left|R\left(\frac{\partial}{\partial z_{\alpha}}, \frac{\partial}{\partial \bar{z}_{\beta}}, \frac{\partial}{\partial z_{\gamma}}, \frac{\partial}{\partial \bar{z}_{\delta}}\right)\right| \\
& \leq \sum_{\alpha, \beta, \gamma, \delta=1}^{n}\left|\frac{\partial^{2}}{\partial z_{\alpha} \partial \bar{z}_{\beta}} \Psi^{*} g\left(\frac{\partial}{\partial z_{\gamma}}, \frac{\partial}{\partial \bar{z}_{\delta}}\right)\right|+C \sum_{\alpha, \gamma, \delta=1}^{n}\left|\frac{\partial}{\partial z_{\alpha}} \Psi^{*} g\left(\frac{\partial}{\partial z_{\gamma}}, \frac{\partial}{\partial \bar{z}_{\delta}}\right)\right|^{2} \\
& \leq C+C\left|z_{n}\right|^{\frac{1}{\beta}-2}+C\left|z_{n}\right|^{\frac{2}{\beta}-4} .
\end{aligned}
$$


This completes the proof.

A similar argument gives a bound for the covariant derivative of the curvature tensor.

Proposition 3.5. We have

$$
|D R|_{g} \leq C+C\left|z_{n}\right|^{\frac{1}{\beta}-3}+C\left|z_{n}\right|^{\frac{2}{\beta}-5} \text {. }
$$

In particular, if $\beta \in\left(0, \frac{1}{2}\right)$, then $|D R|_{g} \leq C\left|z_{n}\right|^{\frac{\varepsilon}{\beta}-1}$ for some $\varepsilon>0$.

Proof. It follows from Lemma 3.2 and Lemma 3.3 that

$$
\sum_{\alpha, \gamma, \delta=1}^{n}\left|\frac{\partial}{\partial z_{\alpha}} \Psi^{*} g\left(\frac{\partial}{\partial z_{\gamma}}, \frac{\partial}{\partial \bar{z}_{\delta}}\right)\right| \leq C+C\left|z_{n}\right|^{\frac{1}{\beta}-2}
$$

and

$$
\sum_{\alpha, \beta, \gamma, \delta=1}^{n}\left|\frac{\partial^{2}}{\partial z_{\alpha} \partial \bar{z}_{\beta}} \Psi^{*} g\left(\frac{\partial}{\partial z_{\gamma}}, \frac{\partial}{\partial \bar{z}_{\delta}}\right)\right| \leq C+C\left|z_{n}\right|^{\frac{1}{\beta}-2}+C\left|z_{n}\right|^{\frac{2}{\beta}-4} .
$$

A similar calculation gives

$$
\sum_{\alpha, \beta, \gamma, \delta=1}^{n}\left|\frac{\partial^{2}}{\partial z_{\alpha} \partial z_{\beta}} \Psi^{*} g\left(\frac{\partial}{\partial z_{\gamma}}, \frac{\partial}{\partial \bar{z}_{\delta}}\right)\right| \leq C+C\left|z_{n}\right|^{\frac{1}{\beta}-3}
$$

and

$$
\sum_{\alpha, \beta, \gamma, \delta, \mu=1}^{n}\left|\frac{\partial^{3}}{\partial z_{\alpha} \partial \bar{z}_{\beta} \partial z_{\mu}} \Psi^{*} g\left(\frac{\partial}{\partial z_{\gamma}}, \frac{\partial}{\partial \bar{z}_{\delta}}\right)\right| \leq C+C\left|z_{n}\right|^{\frac{1}{\beta}-3}+C\left|z_{n}\right|^{\frac{2}{\beta}-5}
$$

Since the metric $\Psi^{*} g$ is uniformly equivalent to the Euclidean metric, we conclude that

$$
\begin{aligned}
|D R|_{g} & \leq C \sum_{\alpha, \beta, \gamma, \delta, \mu=1}^{n}\left|\frac{\partial^{3}}{\partial z_{\alpha} \partial \bar{z}_{\beta} \partial z_{\mu}} \Psi^{*} g\left(\frac{\partial}{\partial z_{\gamma}}, \frac{\partial}{\partial \bar{z}_{\delta}}\right)\right| \\
& +C\left(\sum_{\alpha, \gamma, \delta=1}^{n}\left|\frac{\partial}{\partial z_{\alpha}} \Psi^{*} g\left(\frac{\partial}{\partial z_{\gamma}}, \frac{\partial}{\partial \bar{z}_{\delta}}\right)\right|\right)\left(\sum_{\alpha, \beta, \gamma, \delta=1}^{n}\left|\frac{\partial^{2}}{\partial z_{\alpha} \partial z_{\beta}} \Psi^{*} g\left(\frac{\partial}{\partial z_{\gamma}}, \frac{\partial}{\partial \bar{z}_{\delta}}\right)\right|\right) \\
& +C\left(\sum_{\alpha, \gamma, \delta=1}^{n}\left|\frac{\partial}{\partial z_{\alpha}} \Psi^{*} g\left(\frac{\partial}{\partial z_{\gamma}}, \frac{\partial}{\partial \bar{z}_{\delta}}\right)\right|\right)\left(\sum_{\alpha, \beta, \gamma, \delta=1}^{n}\left|\frac{\partial^{2}}{\partial z_{\alpha} \partial \bar{z}_{\beta}} \Psi^{*} g\left(\frac{\partial}{\partial z_{\gamma}}, \frac{\partial}{\partial \bar{z}_{\delta}}\right)\right|\right) \\
& +C\left(\sum_{\alpha, \gamma, \delta=1}^{n}\left|\frac{\partial}{\partial z_{\alpha}} \Psi^{*} g\left(\frac{\partial}{\partial z_{\gamma}}, \frac{\partial}{\partial \bar{z}_{\delta}}\right)\right|\right)^{3} \\
& \leq C+C\left|z_{n}\right|^{\frac{1}{\beta}-3}+C\left|z_{n}\right|^{\frac{2}{\beta}-5} .
\end{aligned}
$$

This completes the proof.

We next describe a Sobolev inequality for the background edge manifold $(M \backslash \Sigma, g)$. This inequality will be used in the proof of the $C^{0}$ estimate. 
Proposition 3.6. Let $v$ be a smooth function on $M \backslash \Sigma$ satisfying $\sup _{M \backslash \Sigma}|v|<$ $\infty$. Then

$$
\left(\int_{M}|v|^{\frac{2 n}{n-1}} \omega^{n}\right)^{\frac{n-1}{n}} \leq C \int_{M}|d v|_{g}^{2} \omega^{n}+C \int_{M}|v|^{2} \omega^{n}
$$

for some uniform constant $C$.

Proof. We first consider the case when $v$ is supported in a coordinate neighborhood of some point in $\Sigma$. To that end, we fix a point on $\Sigma$, and let $\left(z_{1}, \ldots, z_{n-1}, \zeta\right)$ be complex coordinates around that point. Let $g$ denote the Riemannian metric associated with the Kähler form $\omega$. Then the pull-back of the metric $g$ under the map $\Phi:\left(z_{1}, \ldots, z_{n-1}, \xi\right) \mapsto\left(z_{1}, \ldots, z_{n-1},|\xi|^{\frac{1}{\beta}-1} \xi\right)$ is uniformly equivalent to the Euclidean metric. Therefore, the Sobolev inequality (11) holds if the function $v$ is suported in that coordinate chart. The general case follows in the standard way by using a partition of unity.

Finally, the Fredholm alternative established on page 20 of Donaldson's paper gives:

Theorem 3.7 (S. Donaldson [3]). Let $M$ be a compact Kähler manifold, and let $\Sigma$ be a complex hypersurface in $M$. Moreover, let $\omega$ be the background Kähler metric constructed above, and let $\hat{\omega}$ be a Kähler metric of class $\mathcal{C}^{,}, \alpha$ which is uniformly equivalent to the background metric $\omega$. Then the operator

$$
\mathcal{C}^{2, \alpha, \beta} \rightarrow \mathcal{C}^{, \alpha, \beta}, \quad v \mapsto \Delta_{\hat{\omega}} v
$$

is Fredholm with Fredholm index zero.

\section{A $C^{0}$-Estimate For solutions of $\left(\star_{t}\right)$}

In this section, we establish a uniform $C^{0}$-estimate for solutions of $\left(\star_{t}\right)$. Throughout this section, we consider a pair $(u, c) \in \mathcal{C}^{2, \alpha, \beta} \times \mathbb{R}$ which satisfies the equation

$$
(\omega+\sqrt{-1} \partial \bar{\partial} u)^{n}=e^{t F-c} \omega^{n}
$$

for some $t \in[0,1]$. Since $u \in \mathcal{C}^{2, \alpha, \beta}$, it follows from standard elliptic regularity theory that $u \in C^{\infty}(M \backslash \Sigma)$. For abbreviation, let $\hat{\omega}=\omega+\sqrt{-1} \partial \bar{\partial} u$.

Note that the function $u$ is only defined up to constants. We may normalize $u$ such that

$$
\int_{M} u \omega_{0}^{n}=0
$$

In the first step, we show that the function $u$ is uniformly bounded from above. To that end, we denote by $\Gamma_{p}: M \backslash\{p\} \rightarrow \mathbb{R}$ the Green's function associated with the operator $\Delta_{\omega_{0}}$ with pole at $p$. We may assume that the function $\Gamma_{p}$ is negative everywhere. 
Proposition 4.1. We have

$$
u(p) \leq-n \int_{M} \Gamma_{p} \omega_{0}^{n}+\frac{\int_{M} \lambda|s|_{h}^{2 \beta} \omega_{0}^{n}}{\int_{M} \omega_{0}^{n}}
$$

for all points $p \in M \backslash \Sigma$. In particular, we can find a uniform constant $N$ such that $N-u \geq 1$.

Proof. For abbreviation, let $v=u+\lambda|s|_{h}^{2 \beta}$. Let us fix a point $p \in$ $M \backslash \Sigma$, and let $\chi: M \rightarrow[0,1]$ be a smooth function which vanishes in a neighborhood of $\Sigma$. Using Green's formula, we obtain

$$
\begin{aligned}
\chi(p) v(p) & =\int_{M} \Gamma_{p} \Delta_{\omega_{0}}(\chi v) \omega_{0}^{n}+\frac{\int_{M} \chi v \omega_{0}^{n}}{\int_{M} \omega_{0}^{n}} \\
& =-\int_{M} \Gamma_{p} v \Delta_{\omega_{0}} \chi \omega_{0}^{n}-2 \int_{M} v\left\langle d \chi, d \Gamma_{p}\right\rangle_{g_{0}} \omega_{0}^{n} \\
& +\int_{M} \Gamma_{p} \chi \Delta_{\omega_{0}} v \omega_{0}^{n}+\frac{\int_{M} \chi v \omega_{0}^{n}}{\int_{M} \omega_{0}^{n}} .
\end{aligned}
$$

Using the identity $\omega_{0}+\sqrt{-1} \partial \bar{\partial} v=\hat{\omega}$, we obtain $n+\Delta_{\omega_{0}} v \geq 0$. This implies

$$
\begin{aligned}
\chi(p) v(p) & \leq-\int_{M} \Gamma_{p} v \Delta_{\omega_{0}} \chi \omega_{0}^{n}-2 \int_{M} v\left\langle d \chi, d \Gamma_{p}\right\rangle_{g_{0}} \omega_{0}^{n} \\
& -n \int_{M} \Gamma_{p} \chi \omega_{0}^{n}+\frac{\int_{M} \chi v \omega_{0}^{n}}{\int_{M} \omega_{0}^{n}} .
\end{aligned}
$$

We now choose the cutoff function $\chi$ such that $\chi=1$ outside a small neighborhood of $\Sigma$. We can choose $\chi$ in such a way that $\int_{M}\left(\left|\Delta_{\omega_{0}} \chi\right|+|d \chi|_{g_{0}}\right) \omega_{0}^{n}$ is arbitrarily small. Passing to the limit, we obtain

$$
v(p) \leq-n \int_{M} \Gamma_{p} \omega_{0}^{n}+\frac{\int_{M} v \omega_{0}^{n}}{\int_{M} \omega_{0}^{n}} .
$$

Since $u(p) \leq v(p)$ and $\int_{M} u \omega_{0}^{n}=0$, the assertion follows.

Lemma 4.2. We have

$$
\int_{M}(N-u) \omega^{n} \leq C
$$

for some uniform constant $C$.

Proof. Since $u \in \mathcal{C}^{2, \alpha, \beta}$, we have $\sup _{M \backslash \Sigma}|d u|_{g}<\infty$. This implies $\sup _{M \backslash \Sigma}|s|_{h}^{1-\beta}|d u|_{g_{0}}<\infty$. Hence, integration by parts gives

$$
\begin{aligned}
& \int_{\left\{|s|_{h} \geq r\right\}}(N-u) \Delta_{\omega_{0}}\left(|s|_{h}^{2 \beta}\right) \omega_{0}^{n} \\
& =-\int_{\left\{|s|_{h} \geq r\right\}}|s|_{h}^{2 \beta} \Delta_{\omega_{0}} u \omega_{0}^{n}+O\left(r^{2 \beta}\right) .
\end{aligned}
$$


Using the inequality $n+\Delta_{\omega_{0}}\left(u+\lambda|s|_{h}^{2 \beta}\right) \geq 0$, we obtain

$$
\begin{aligned}
& \int_{\left\{|s|_{h} \geq r\right\}}(N-u) \Delta_{\omega_{0}}\left(|s|_{h}^{2 \beta}\right) \omega_{0}^{n} \\
& \leq \int_{\left\{|s|_{h} \geq r\right\}}|s|_{h}^{2 \beta}\left(n+\lambda \Delta_{\omega_{0}}\left(|s|_{h}^{2 \beta}\right)\right) \omega_{0}^{n}+O\left(r^{2 \beta}\right) .
\end{aligned}
$$

Taking the limit as $r \rightarrow 0$ gives

$$
\int_{M}(N-u) \Delta_{\omega_{0}}\left(|s|_{h}^{2 \beta}\right) \omega_{0}^{n} \leq \int_{M}|s|_{h}^{2 \beta}\left(n+\lambda \Delta_{\omega_{0}}\left(|s|_{h}^{2 \beta}\right)\right) \omega_{0}^{n} \leq C .
$$

We next observe that

$$
\Delta_{\omega_{0}}\left(|s|_{h}^{2 \beta}\right) \geq \delta|s|_{h}^{2 \beta-2}-C_{0}
$$

for suitable constants $\delta, C_{0}>0$. Since $N-u$ is a positive function, we conclude that

$$
\int_{M}(N-u)\left(\delta|s|_{h}^{2 \beta-2}-C_{0}\right) \omega_{0}^{n} \leq C .
$$

Using the normalization (12), we obtain

$$
\int_{M}(N-u)|s|_{h}^{2 \beta-2} \omega_{0}^{n} \leq C
$$

hence

$$
\int_{M}(N-u) \omega^{n} \leq C
$$

This completes the proof.

We next establish upper and lower bounds for the number $c$.

Lemma 4.3. We have

$$
\int_{M}\left(e^{t F-c}-1\right) \omega^{n}=0
$$

In particular, $\inf _{M} t F \leq c \leq \sup _{M} t F$.

Proof. Let $\chi: M \rightarrow[0,1]$ be a smooth cutoff function which vanishes in a neighborhood of $\Sigma$. Then

$$
\begin{aligned}
\int_{M} \chi\left(e^{t F-c}-1\right) \omega^{n} & =\int_{M} \chi\left(\hat{\omega}^{n}-\omega^{n}\right) \\
& =\sum_{m=0}^{n-1} \int_{M} \chi(\hat{\omega}-\omega) \wedge \omega^{m} \wedge \hat{\omega}^{n-m-1} \\
& =\sum_{m=0}^{n-1} \int_{M} \chi \sqrt{-1} \partial \bar{\partial} u \wedge \omega^{m} \wedge \hat{\omega}^{n-m-1} \\
& =-\sum_{m=0}^{n-1} \int_{M} \sqrt{-1} \partial \chi \wedge \bar{\partial} u \wedge \omega^{m} \wedge \hat{\omega}^{n-m-1}
\end{aligned}
$$


Note that $\sup _{M \backslash \Sigma}|d u|_{g}<\infty$ and $\sup _{M \backslash \Sigma}|\partial \bar{\partial} u|_{g}<\infty$ since $u \in \mathcal{C}^{2, \alpha, \beta}$. We now choose $\chi$ such that $\chi=1$ outside a small neighborhood of $\Sigma$. Moreover, we can arrange for $\int_{M}|d \chi|_{g} \omega^{n}$ to be arbitrarily small. Passing to the limit, the assertion follows.

In the next step, we establish an upper bound for the function $N-u$. This follows from an adaptation of the arguments in [10, Section 5.1.

Lemma 4.4. For each $p>1$, we have

$$
\frac{4(p-1)}{p^{2}} \int_{M}\left|d\left((N-u)^{p / 2}\right)\right|_{g}^{2} \omega^{n} \leq e^{\sup _{M}(t F)-\inf _{M}(t F)} \int_{M}(N-u)^{p} \omega^{n}
$$

Proof. Let $\chi: M \rightarrow[0,1]$ be a smooth cutoff function which vanishes in a neighborhood of $\Sigma$. Then

$$
\begin{aligned}
& \int_{M} \chi(N-u)^{p-1}\left(e^{t F-c}-1\right) \omega^{n} \\
& =\int_{M} \chi(N-u)^{p-1}\left(\hat{\omega}^{n}-\omega^{n}\right) \\
& =\sum_{m=0}^{n-1} \int_{M} \chi(N-u)^{p-1}(\hat{\omega}-\omega) \wedge \omega^{m} \wedge \hat{\omega}^{n-m-1} \\
& =\sum_{m=0}^{n-1} \int_{M} \chi(N-u)^{p-1} \sqrt{-1} \partial \bar{\partial} u \wedge \omega^{m} \wedge \hat{\omega}^{n-m-1} \\
& =-\sum_{m=0}^{n-1} \int_{M} \sqrt{-1} \partial\left(\chi(N-u)^{p-1}\right) \wedge \bar{\partial} u \wedge \omega^{m} \wedge \hat{\omega}^{n-m-1} \\
& =(p-1) \sum_{m=0}^{n-1} \int_{M} \chi(N-u)^{p-2} \sqrt{-1} \partial u \wedge \bar{\partial} u \wedge \omega^{m} \wedge \hat{\omega}^{n-m-1} \\
& -\sum_{m=0}^{n-1} \int_{M}(N-u)^{p-1} \sqrt{-1} \partial \chi \wedge \bar{\partial} u \wedge \omega^{m} \wedge \hat{\omega}^{n-m-1} .
\end{aligned}
$$

Note that $\sup _{M \backslash \Sigma}|d u|_{g}<\infty$ and $\sup _{M \backslash \Sigma}|\partial \bar{\partial} u|_{g}<\infty$ since $u \in \mathcal{C}^{2, \alpha, \beta}$. As above, we may choose $\chi$ such that $\chi=1$ outside a small neighborhood of $\Sigma$ and $\int_{M}|d \chi|_{g} \omega^{n}$ is arbitrarily small. Passing to the limit, we obtain

$$
\begin{aligned}
& \int_{M}(N-u)^{p-1}\left(e^{t F-c}-1\right) \omega^{n} \\
& =(p-1) \sum_{m=0}^{n-1} \int_{M}(N-u)^{p-2} \sqrt{-1} \partial u \wedge \bar{\partial} u \wedge \omega^{m} \wedge \hat{\omega}^{n-m-1} .
\end{aligned}
$$


This implies

$$
\begin{aligned}
& \int_{M}(N-u)^{p-1}\left(e^{t F-c}-1\right) \omega^{n} \\
& \geq(p-1) \int_{M}(N-u)^{p-2} \sqrt{-1} \partial u \wedge \bar{\partial} u \wedge \omega^{n-1} \\
& =(p-1) \int_{M}(N-u)^{p-2}|d u|_{g}^{2} \omega^{n} \\
& =\frac{4(p-1)}{p^{2}} \int_{M}\left|d\left((N-u)^{p / 2}\right)\right|_{g}^{2} \omega^{n}
\end{aligned}
$$

On the other hand, we have $c \geq \inf _{M}(t F)$ by Lemma 4.3. From this, we deduce that

$$
\begin{aligned}
\int_{M}(N-u)^{p-1}\left(e^{t F-c}-1\right) \omega^{n} & \leq e^{\sup _{M}(t F)-\inf _{M}(t F)} \int_{M}(N-u)^{p-1} \omega^{n} \\
& \leq e^{\sup _{M}(t F)-\inf _{M}(t F)} \int_{M}(N-u)^{p} \omega^{n} .
\end{aligned}
$$

Putting these facts together, the assertion follows.

Lemma 4.5. We have

$$
\int_{M}(N-u)^{\frac{2 n}{n-1}} \omega^{n} \leq C
$$

for some uniform constant $C$.

Proof. Applying Lemma 4.4 with $p=2$ gives

$$
\int_{M}|d(N-u)|_{g}^{2} \omega^{n} \leq e^{\sup _{M}(t F)-\inf _{M}(t F)} \int_{M}(N-u)^{2} \omega^{n} .
$$

Moreover, we have

$$
\left(\int_{M}(N-u)^{\frac{2 n}{n-1}} \omega^{n}\right)^{\frac{n-1}{n}} \leq C \int_{M}|d(N-u)|_{g}^{2} \omega^{n}+C \int_{M}(N-u)^{2} \omega^{n}
$$

by Proposition 3.6. Putting these facts together, we obtain

$$
\begin{aligned}
& \left(\int_{M}(N-u)^{\frac{2 n}{n-1}} \omega^{n}\right)^{\frac{n-1}{n}} \\
& \leq C \int_{M}(N-u)^{2} \omega^{n} \leq C\left(\int_{M}(N-u) \omega^{n}\right)^{\frac{2}{n+1}}\left(\int_{M}(N-u)^{\frac{2 n}{n-1}} \omega^{n}\right)^{\frac{n-1}{n+1}}
\end{aligned}
$$

for some uniform constant $C$. Thus, we conclude that

$$
\left(\int_{M}(N-u)^{\frac{2 n}{n-1}} \omega^{n}\right)^{\frac{n-1}{2 n}} \leq C \int_{M}(N-u) \omega^{n}
$$

Hence, the assertion follows from Lemma 4.2. 
Proposition 4.6. We have $\sup _{M}(N-u) \leq C$ for some uniform constant $C$.

Proof. By Lemma 4.5, we have

$$
\int_{M}(N-u)^{\frac{2 n}{n-1}} \omega^{n} \leq C .
$$

Moreover, using Proposition 3.6 and Lemma 4.4, we obtain

$$
\begin{aligned}
& \left(\int_{M}(N-u)^{\frac{p n}{n-1}} \omega^{n}\right)^{\frac{n-1}{n}} \\
& \leq C \int_{M}\left|d\left((N-u)^{p / 2}\right)\right|^{2} \omega^{n}+C \int_{M}(N-u)^{p} \omega^{n} \\
& \leq C\left(\frac{p^{2}}{4(p-1)} e^{\sup _{M}(t F)-\inf _{M}(t F)}+1\right) \int_{M}(N-u)^{p} \omega^{n}
\end{aligned}
$$

for each $p>1$. Hence, it follows from the standard Moser iteration technique that $\sup _{M}(N-u) \leq C$ for some uniform constant $C$.

Combining Proposition 4.1 and Proposition 4.6, we conclude that $\sup _{M}|u| \leq$ $C$ for some uniform constant $C$.

\section{An ESTIMATE FOR $\partial \bar{\partial} u$}

In this section, we establish a uniform estimate for the Laplacian $\Delta_{\omega} u$. The following lemma will be useful:

Lemma 5.1. If $\varepsilon>0$ is sufficiently small, then

$$
\sqrt{-1} \partial \bar{\partial}\left(|s|_{h}^{2 \varepsilon}\right)+\omega \geq \varepsilon^{2}|s|_{h}^{2 \varepsilon} \sqrt{-1} \partial \log \left(|s|_{h}^{2}\right) \wedge \bar{\partial} \log \left(|s|_{h}^{2}\right) \geq 0 .
$$

Proof. We can find a positive constant $C$ such that

$$
\sqrt{-1} \partial \bar{\partial} \log \left(|s|_{h}^{2}\right)+C \omega_{0} \geq 0 .
$$

This implies

$$
\sqrt{-1} \partial \bar{\partial}\left(|s|_{h}^{2 \varepsilon}\right)+C \varepsilon|s|_{h}^{2 \varepsilon} \omega_{0} \geq \varepsilon^{2}|s|_{h}^{2 \varepsilon} \sqrt{-1} \partial \log \left(|s|_{h}^{2}\right) \wedge \bar{\partial} \log \left(|s|_{h}^{2}\right) .
$$

If we choose $\varepsilon$ sufficiently small, then $C \varepsilon \omega_{0} \leq \omega$. From this, the assertion follows.

The proof of the Laplacian estimate largely follows the arguments in Yau's paper [15]. There is an additional technical issue in that function $e^{-L u}(n+$ $\left.\Delta_{\omega} u\right)$ may attain its maximum on the singular set $\Sigma$. This obstacle can be overcome using a trick due to T. Jeffres (cf. [6], Section 4). Jeffres studied the case of Kähler-Einstein metrics with negative scalar curvature, and proved a $C^{0}$ estimate in that setting. A similar trick works for the Laplacian estimate.

Proposition 5.2. Let $u \in \mathcal{C}^{2, \alpha, \beta}$ be a solution of $\left(\star_{t}\right)$ for some $t \in[0,1]$. Then $\Delta_{\omega} u \leq C$ for some uniform constant $C$. 
Proof. Let us fix a point $q \in M \backslash \Sigma$. We may choose complex coordinates around $q$ so that $\omega_{\mu \bar{\nu}}=\delta_{\mu \nu}$ and $u_{\mu \bar{\nu}}=u_{\mu \bar{\mu}} \delta_{\mu \nu}$. By Proposition 3.4, the curvature tensor of the background metric $\omega$ is uniformly bounded. Hence, we can find a positive constant $L$ such that

$$
L+\inf _{\mu \neq \nu} R_{\mu \bar{\mu} \nu \bar{\nu}} \geq 2 .
$$

Using equation (2.18) in [15], we obtain

$$
\begin{aligned}
& \Delta_{\hat{\omega}}\left(e^{-L u}\left(n+\Delta_{\omega} u\right)\right) \\
& \geq e^{-L u}\left(\Delta_{\omega}(t F)-n^{2} \inf _{\mu \neq \nu} R_{\mu \bar{\mu} \nu \bar{\nu}}\right)-n L e^{-L u}\left(n+\Delta_{\omega} u\right) \\
& +\left(L+\inf _{\mu \neq \nu} R_{\mu \bar{\mu} \nu \bar{\nu}}\right) e^{-L u}\left(n+\Delta_{\omega} u\right) \sum_{\mu=1}^{n} \frac{1}{1+u_{\mu \bar{\mu}}}
\end{aligned}
$$

at the point $q$. By Proposition 3.1, we have $\left|\Delta_{\omega} F\right| \leq C$. Moreover, Proposition 3.4 implies that the background metric $\omega$ has bounded curvature. This implies

$$
\begin{aligned}
\Delta_{\hat{\omega}}\left(e^{-L u}\left(n+\Delta_{\omega} u\right)\right) & \geq 2 e^{-L u}\left(n+\Delta_{\omega} u\right) \sum_{\mu=1}^{n} \frac{1}{1+u_{\mu \bar{\mu}}} \\
& -n L e^{-L u}\left(n+\Delta_{\omega} u\right)-e^{-L u} C
\end{aligned}
$$

at the point $q$. We next observe that

$$
n+\Delta_{\omega} u \geq n\left(\prod_{\mu=1}^{n}\left(1+u_{\mu \bar{\mu}}\right)\right)^{\frac{1}{n}}=n e^{\frac{t F-c}{n}}
$$

and

$$
\sum_{\mu=1}^{n} \frac{1}{1+u_{\mu \bar{\mu}}} \geq\left(\frac{n+\Delta_{\omega} u}{\prod_{\mu=1}^{n}\left(1+u_{\mu \bar{\mu}}\right)}\right)^{\frac{1}{n-1}}=e^{-\frac{t F-c}{n-1}}\left(n+\Delta_{\omega} u\right)^{\frac{1}{n-1}}
$$

(cf. [15], equation (2.19)). Thus, we conclude that

$$
\begin{aligned}
& \Delta_{\hat{\omega}}\left(e^{-L u}\left(n+\Delta_{\omega} u\right)\right) \\
& \geq n e^{\frac{t F-c}{n}} e^{-L u} \sum_{\mu=1}^{n} \frac{1}{1+u_{\mu \bar{\mu}}}+e^{-\frac{t F-c}{n-1}} e^{-L u}\left(n+\Delta_{\omega} u\right)^{\frac{n}{n-1}} \\
& -n L e^{-L u}\left(n+\Delta_{\omega} u\right)-e^{-L u} C .
\end{aligned}
$$

Moreover, it follows from Propositions 4.1 and 4.6 that $|u| \leq C$ for some uniform constant $C$. Putting these facts together, we obtain

$$
\begin{aligned}
& \Delta_{\hat{\omega}}\left(e^{-L u}\left(n+\Delta_{\omega} u\right)\right) \\
& \geq \kappa \sum_{\mu=1}^{n} \frac{1}{1+u_{\mu \bar{\mu}}}+\kappa e^{-\frac{n L u}{n-1}}\left(n+\Delta_{\omega} u\right)^{\frac{n}{n-1}}-n L e^{-L u}\left(n+\Delta_{\omega} u\right)-C
\end{aligned}
$$

for uniform constants $\kappa, C>0$. 
Let us fix $\varepsilon>0$ sufficiently small. By Lemma 5.1, we have

$$
\sqrt{-1} \partial \bar{\partial}\left(|s|_{h}^{2 \varepsilon}\right)+\omega \geq 0
$$

hence

$$
\Delta_{\hat{\omega}}\left(|s|_{h}^{2 \varepsilon}\right)+\sum_{\mu=1}^{n} \frac{1}{1+u_{\mu \bar{\mu}}} \geq 0 .
$$

Consequently, the function

$$
H=e^{-L u}\left(n+\Delta_{\omega} u\right)+\kappa|s|_{h}^{2 \varepsilon}
$$

satisfies

$$
\begin{aligned}
\Delta_{\hat{\omega}} H & \geq \kappa \Delta_{\hat{\omega}}\left(|s|_{h}^{2 \varepsilon}\right)+\kappa \sum_{\mu=1}^{n} \frac{1}{1+u_{\mu \bar{\mu}}}+\kappa\left(H-\kappa|s|_{h}^{2 \varepsilon}\right)^{\frac{n}{n-1}} \\
& -n L\left(H-\kappa|s|_{h}^{2 \varepsilon}\right)-C \\
& \geq \kappa\left(H-\kappa|s|_{h}^{2 \varepsilon}\right)^{\frac{n}{n-1}}-n L\left(H-\kappa|s|_{h}^{2 \varepsilon}\right)-C .
\end{aligned}
$$

Since the function $u$ is of class $\mathcal{C}^{2, \alpha, \beta}$, the function $\Delta_{\omega} u$ is of class $\mathcal{C}^{, \alpha, \beta}$. Consequently, if $\varepsilon>0$ is small, then the function $H$ attains its maximum at some point $q \in M \backslash \Sigma$. The maximum principle then implies that $\sup _{M \backslash \Sigma} H \leq C$ for some constant $C$. Consequently, the function $\Delta_{\omega} u$ is uniformly bounded from above, as claimed.

It follows from $\left(\star_{t}\right)$ that the function $\frac{\hat{\omega}^{n}}{\omega^{n}}$ is uniformly bounded from above and below. Using Proposition 5.2, we conclude that $a_{1} \omega \leq \hat{\omega} \leq a_{2} \omega$ for uniform constants $a_{1}, a_{2}>0$.

\section{An estimate For the COVARIANt DeRIVAtive of $\partial \bar{\partial} u$}

As above, we consider a pair $(u, c) \in \mathcal{C}^{2, \alpha, \beta} \times \mathbb{R}$ which satisfies the equation

$$
(\omega+\sqrt{-1} \partial \bar{\partial} u)^{n}=e^{t F-c} \omega^{n}
$$

for some $t \in[0,1]$. Moreover, we let $\hat{\omega}=\omega+\sqrt{-1} \partial \bar{\partial} u$.

Our goal in this section is to establish a uniform bound for the covariant derivative of $\partial \bar{\partial} u$. This estimate will be proved using the maximum principle. However, in order to apply the maximum principle, it is necessary to analyze the behavior of the third derivatives of $u$ near $\Sigma$. To that end, we fix a point $p \in \Sigma$. Moreover, let $\left(z_{1}, \ldots, z_{n-1}, \zeta\right)$ be complex coordinates around $p$. 
Lemma 6.1. We have

$$
\begin{gathered}
\frac{\partial^{3} u}{\partial z_{i} \partial \bar{z}_{j} \partial z_{k}} \in \mathcal{C}^{, \alpha, \beta} \\
|\zeta|^{1-\beta} \frac{\partial^{3} u}{\partial z_{i} \partial \bar{\zeta} \partial z_{k}} \in \mathcal{C}_{0}^{, \alpha, \beta} \\
|\zeta|^{1-\beta} \frac{\partial^{3} u}{\partial \zeta \partial \bar{z}_{j} \partial z_{k}} \in \mathcal{C}_{0}^{, \alpha, \beta} \\
|\zeta|^{2-2 \beta} \frac{\partial^{3} u}{\partial \zeta \partial \bar{\zeta} \partial z_{k}} \in \mathcal{C}^{, \alpha, \beta}
\end{gathered}
$$

for all $i, j, k \in\{1, \ldots, n-1\}$.

Proof. Let us fix an integer $k \in\{1, \ldots, n-1\}$. Using the formula

$$
\omega=\omega_{0}+\lambda \sqrt{-1} \partial \bar{\partial}\left(\rho|\zeta|^{2 \beta}\right)
$$

we obtain

$$
\frac{\partial}{\partial z_{k}} \omega=\frac{\partial}{\partial z_{k}} \omega_{0}+\lambda \sqrt{-1} \partial \bar{\partial}\left(\frac{\partial \rho}{\partial z_{k}}|\zeta|^{2 \beta}\right) .
$$

Hence, if $\alpha>0$ is sufficiently small, then the $(1,1)$-form $\frac{\partial}{\partial z_{k}} \omega$ is of class $\mathcal{C}^{,}, \alpha, \beta$. This implies $\operatorname{tr}_{\omega}\left(\frac{\partial}{\partial z_{k}} \omega\right) \in \mathcal{C}^{, \alpha, \beta}$. Since $\hat{\omega}$ is uniformly equivalent to $\omega$ and of class $\mathcal{C}^{, \alpha, \beta}$, we also have $\operatorname{tr}_{\hat{\omega}}\left(\frac{\partial}{\partial z_{k}} \omega\right) \in \mathcal{C}^{, \alpha, \beta}$. Moreover, it follows from (8) that $\frac{\partial}{\partial z_{k}} F \in \mathcal{C}, \alpha, \beta$. We now define

$$
v=\frac{\partial}{\partial z_{k}} u \in \mathcal{C}^{, \alpha, \beta}
$$

and

$$
f=\operatorname{tr}_{\omega}\left(\frac{\partial}{\partial z_{k}} \omega\right)-\operatorname{tr}_{\hat{\omega}}\left(\frac{\partial}{\partial z_{k}} \omega\right)+\frac{\partial}{\partial z_{k}}(t F) \in \mathcal{C}^{, \alpha, \beta} .
$$

Differentiating the equation $\left(\star_{t}\right)$ with respect to $z_{k}$, we conclude that $\Delta_{\hat{\omega}} v=$ $f$ away from $\Sigma$. Hence, Corollary 2.6 implies that $v \in \mathcal{C}^{2, \alpha, \beta}$. From this, the assertion follows.

Lemma 6.2. We have

$$
|\zeta|^{2-2 \beta}\left(\frac{\partial^{3} u}{\partial \zeta \partial \bar{z}_{j} \partial \zeta}+\frac{1-\beta}{\zeta} \frac{\partial^{2} u}{\partial \zeta \partial \bar{z}_{j}}\right)=O\left(|\zeta|^{\alpha \beta}\right)
$$

for all $j \in\{1, \ldots, n-1\}$.

Proof. Let us fix an integer $j \in\{1, \ldots, n-1\}$ and let $v=\frac{\partial}{\partial \bar{z}_{j}} u$. By Lemma 6.1, we have

$$
|\zeta|^{2-2 \beta} \frac{\partial^{2} v}{\partial \zeta \partial \bar{\zeta}} \in \mathcal{C}^{, \alpha, \beta}
$$


Applying Proposition A.1 to the function $\zeta \mapsto v\left(z_{1}, \ldots, z_{n-1}, \zeta\right)$, we conclude that

$$
|\zeta|^{2-2 \beta}\left(\frac{\partial^{2} v}{\partial \zeta \partial \zeta}+\frac{1-\beta}{\zeta} \frac{\partial v}{\partial \zeta}\right)=O\left(|\zeta|^{\alpha \beta}\right)
$$

as claimed.

In the next step, we study the third order covariant derivatives of $u$ with respect to the background edge metric $\omega$. We first analyze the Christoffel symbols of the background metric $g$.

Lemma 6.3. The Christoffel symbols of $g$ satisfy

$$
\Gamma_{i k}^{j} \in \mathcal{C}^{, \alpha, \beta}, \quad|\zeta|^{1-\beta} \Gamma_{i \zeta}^{k} \in \mathcal{C}_{0}^{, \alpha, \beta}, \quad|\zeta|^{\beta-1} \Gamma_{i k}^{\zeta} \in \mathcal{C}_{0}^{, \alpha, \beta}
$$

and

$$
\Gamma_{i \zeta}^{\zeta} \in \mathcal{C}^{, \alpha, \beta}, \quad|\zeta|^{2-2 \beta} \Gamma_{\zeta \zeta}^{k} \in \mathcal{C}_{0}^{, \alpha, \beta}, \quad|\zeta|^{1-\beta}\left(\Gamma_{\zeta \zeta}^{\zeta}+\frac{1-\beta}{\zeta}\right) \in \mathcal{C}_{0}^{, \alpha, \beta}
$$

for all $i, j, k \in\{1, \ldots, n-1\}$.

Proof. Recall that

$$
\begin{aligned}
\omega & =\omega_{0}+\lambda|\zeta|^{2 \beta} \sqrt{-1} \partial \bar{\partial} \rho \\
& +\lambda \beta|\zeta|^{2 \beta-2} \zeta \sqrt{-1} \partial \rho \wedge d \bar{\zeta} \\
& +\lambda \beta|\zeta|^{2 \beta-2} \bar{\zeta} \sqrt{-1} d \zeta \wedge \bar{\partial} \rho \\
& +\lambda \beta^{2} \rho \sqrt{-1}|\zeta|^{2 \beta-2} d \zeta \wedge d \bar{\zeta}
\end{aligned}
$$

Hence, if $\alpha>0$ is sufficiently small, then we have

$$
g^{k \bar{l}} \in \mathcal{C}^{, \alpha, \beta}, \quad|\zeta|^{\beta-1} g^{k \bar{\zeta}} \in \mathcal{C}_{0}^{, \alpha, \beta}, \quad|\zeta|^{2 \beta-2} g^{\zeta \bar{\zeta}} \in \mathcal{C}^{, \alpha, \beta} .
$$

Moreover, since $\beta \in\left(0, \frac{1}{2}\right)$, we have

$$
\begin{aligned}
& \frac{\partial}{\partial z_{i}} g\left(\frac{\partial}{\partial z_{k}}, \frac{\partial}{\partial \bar{z}_{l}}\right) \in \mathcal{C}^{, \alpha, \beta}, \\
& |\zeta|^{1-\beta} \frac{\partial}{\partial z_{i}} g\left(\frac{\partial}{\partial \zeta}, \frac{\partial}{\partial \bar{z}_{l}}\right) \in \mathcal{C}_{0}^{, \alpha, \beta}, \\
& |\zeta|^{2-2 \beta} \frac{\partial}{\partial z_{i}} g\left(\frac{\partial}{\partial \zeta}, \frac{\partial}{\partial \bar{\zeta}}\right) \in \mathcal{C}^{, \alpha, \beta}, \\
& |\zeta|^{2-2 \beta}\left[\frac{\partial}{\partial \zeta} g\left(\frac{\partial}{\partial \zeta}, \frac{\partial}{\partial \bar{z}_{l}}\right)+\frac{1-\beta}{\zeta} g\left(\frac{\partial}{\partial \zeta}, \frac{\partial}{\partial \bar{z}_{l}}\right)\right] \in \mathcal{C}_{0}^{, \alpha, \beta}, \\
& |\zeta|^{3-3 \beta}\left[\frac{\partial}{\partial \zeta} g\left(\frac{\partial}{\partial \zeta}, \frac{\partial}{\partial \bar{\zeta}}\right)+\frac{1-\beta}{\zeta} g\left(\frac{\partial}{\partial \zeta}, \frac{\partial}{\partial \bar{\zeta}}\right)\right] \in \mathcal{C}_{0}^{, \alpha, \beta}
\end{aligned}
$$


if $\alpha>0$ is sufficiently small. This implies

$$
\begin{aligned}
& \Gamma_{i k}^{j}=\sum_{l=1}^{n-1} g^{j \bar{l}} \partial_{i} g_{k \bar{l}}+g^{j \bar{\zeta}} \partial_{i} g_{k \bar{\zeta}} \in \mathcal{C}^{, \alpha, \beta} \\
& |\zeta|^{1-\beta} \Gamma_{i \zeta}^{k}=|\zeta|^{1-\beta} \sum_{l=1}^{n-1} g^{k \bar{l}} \partial_{i} g_{\zeta \bar{l}}+|\zeta|^{1-\beta} g^{k \bar{\zeta}} \partial_{i} g_{\zeta \bar{\zeta}} \in \mathcal{C}_{0}^{, \alpha, \beta} \\
& |\zeta|^{\beta-1} \Gamma_{i k}^{\zeta}=|\zeta|^{\beta-1} \sum_{l=1}^{n-1} g^{\zeta \bar{l}} \partial_{i} g_{k \bar{l}}+|\zeta|^{\beta-1} g^{\zeta \bar{\zeta}} \partial_{i} g_{k \bar{\zeta}} \in \mathcal{C}_{0}^{, \alpha, \beta} \\
& \Gamma_{i \zeta}^{\zeta}=\sum_{l=1}^{n-1} g^{\zeta \bar{l}} \partial_{i} g_{\zeta \bar{l}}+g^{\zeta \bar{\zeta}} \partial_{i} g_{\zeta \bar{\zeta}} \in \mathcal{C}^{, \alpha, \beta}
\end{aligned}
$$

Moreover, we have

$$
\begin{aligned}
|\zeta|^{2-2 \beta} \Gamma_{\zeta \zeta}^{k} & =|\zeta|^{2-2 \beta} \sum_{l=1}^{n-1} g^{k \bar{l}}\left(\partial_{\zeta} g_{\zeta \bar{l}}+\frac{1-\beta}{\zeta} g_{\zeta \bar{l}}\right) \\
& +|\zeta|^{2-2 \beta} g^{k \bar{\zeta}}\left(\partial_{\zeta} g_{\zeta \bar{\zeta}}+\frac{1-\beta}{\zeta} g_{\zeta \bar{\zeta}}\right) \in \mathcal{C}_{0}^{, \alpha, \beta}
\end{aligned}
$$

and

$$
\begin{aligned}
|\zeta|^{1-\beta}\left(\Gamma_{\zeta \zeta}^{\zeta}+\frac{1-\beta}{\zeta}\right) & =|\zeta|^{1-\beta} \sum_{l=1}^{n-1} g^{\zeta \bar{l}}\left(\partial_{\zeta} g_{\zeta \bar{l}}+\frac{1-\beta}{\zeta} g_{\zeta \bar{l}}\right) \\
& +|\zeta|^{1-\beta} g^{\zeta \bar{\zeta}}\left(\partial_{\zeta} g_{\zeta \bar{\zeta}}+\frac{1-\beta}{\zeta} g_{\zeta \bar{\zeta}}\right) \in \mathcal{C}_{0}^{, \alpha, \beta}
\end{aligned}
$$

This completes the proof.

Proposition 6.4. The third order covariant derivatives of u satisfy

$$
\begin{aligned}
& \left(D^{3} u\right)\left(\frac{\partial}{\partial z_{i}}, \frac{\partial}{\partial \bar{z}_{j}}, \frac{\partial}{\partial z_{k}}\right) \in \mathcal{C}^{, \alpha, \beta}, \\
& |\zeta|^{1-\beta}\left(D^{3} u\right)\left(\frac{\partial}{\partial z_{i}}, \frac{\partial}{\partial \bar{\zeta}}, \frac{\partial}{\partial z_{k}}\right) \in \mathcal{C}_{0}^{, \alpha, \beta}, \\
& |\zeta|^{1-\beta}\left(D^{3} u\right)\left(\frac{\partial}{\partial \zeta}, \frac{\partial}{\partial \bar{z}_{j}}, \frac{\partial}{\partial z_{k}}\right) \in \mathcal{C}_{0}^{, \alpha, \beta} \\
& |\zeta|^{2-2 \beta}\left(D^{3} u\right)\left(\frac{\partial}{\partial \zeta}, \frac{\partial}{\partial \bar{\zeta}}, \frac{\partial}{\partial z_{k}}\right) \in \mathcal{C}^{, \alpha, \beta}
\end{aligned}
$$

for all $i, j, k \in\{1, \ldots, n-1\}$. Moreover, we have

$$
|\zeta|^{2-2 \beta}\left(D^{3} u\right)\left(\frac{\partial}{\partial \zeta}, \frac{\partial}{\partial \bar{z}_{j}}, \frac{\partial}{\partial \zeta}\right)=O\left(|\zeta|^{\alpha \beta}\right)
$$

for all $j \in\{1, \ldots, n-1\}$. 
Proof. Using Lemma 6.1 and Lemma 6.3, we obtain

$$
\begin{aligned}
& \left(D^{3} u\right)\left(\frac{\partial}{\partial z_{i}}, \frac{\partial}{\partial \bar{z}_{j}}, \frac{\partial}{\partial z_{k}}\right) \\
& =\frac{\partial^{3} u}{\partial z_{i} \partial \bar{z}_{j} \partial z_{k}}-\sum_{l=1}^{n-1} \Gamma_{i k}^{l} \frac{\partial^{2} u}{\partial z_{l} \partial \bar{z}_{j}}-\Gamma_{i k}^{\zeta} \frac{\partial^{2} u}{\partial \zeta \partial \bar{z}_{j}} \in \mathcal{C}^{, \alpha, \beta} \\
& |\zeta|^{1-\beta}\left(D^{3} u\right)\left(\frac{\partial}{\partial z_{i}}, \frac{\partial}{\partial \bar{\zeta}}, \frac{\partial}{\partial z_{k}}\right) \\
& =|\zeta|^{1-\beta} \frac{\partial^{3} u}{\partial z_{i} \partial \bar{\zeta} \partial z_{k}}-|\zeta|^{1-\beta} \sum_{l=1}^{n-1} \Gamma_{i k}^{l} \frac{\partial^{2} u}{\partial z_{l} \partial \bar{\zeta}}-|\zeta|^{1-\beta} \Gamma_{i k}^{\zeta} \frac{\partial^{2} u}{\partial \zeta \partial \bar{\zeta}_{0}} \in \mathcal{C}_{0}^{, \alpha, \beta} \\
& |\zeta|^{1-\beta}\left(D^{3} u\right)\left(\frac{\partial}{\partial \zeta}, \frac{\partial}{\partial \bar{z}_{j}}, \frac{\partial}{\partial z_{k}}\right) \\
& =|\zeta|^{1-\beta} \frac{\partial^{3} u}{\partial \zeta \partial \bar{z}_{j} \partial z_{k}}-|\zeta|^{1-\beta} \sum_{l=1}^{n-1} \Gamma_{k \zeta}^{l} \frac{\partial^{2} u}{\partial z_{l} \partial \bar{z}_{j}}-|\zeta|^{1-\beta} \Gamma_{k \zeta}^{\zeta} \frac{\partial^{2} u}{\partial \zeta \partial \bar{z}_{j}} \in \mathcal{C}_{0}^{, \alpha, \beta} \\
& |\zeta|^{2-2 \beta}\left(D^{3} u\right)\left(\frac{\partial}{\partial \zeta}, \frac{\partial}{\partial \bar{\zeta}^{\prime}}, \frac{\partial}{\partial z_{k}}\right) \\
& =|\zeta|^{2-2 \beta} \frac{\partial^{3} u}{\partial \zeta \partial \bar{\zeta} \partial z_{k}}-|\zeta|^{2-2 \beta} \sum_{l=1}^{n-1} \Gamma_{k \zeta}^{l} \frac{\partial^{2} u}{\partial z_{l} \partial \bar{\zeta}}-|\zeta|^{2-2 \beta} \Gamma_{k \zeta}^{\zeta} \frac{\partial^{2} u}{\partial \zeta \partial \bar{\zeta}^{\prime}} \in \mathcal{C}^{, \alpha, \beta}
\end{aligned}
$$

We next observe that

$$
\begin{aligned}
|\zeta|^{2-2 \beta}\left(D^{3} u\right)\left(\frac{\partial}{\partial \zeta}, \frac{\partial}{\partial \bar{z}_{j}}, \frac{\partial}{\partial \zeta}\right) & =|\zeta|^{2-2 \beta}\left(\frac{\partial^{3} u}{\partial \zeta \partial \bar{z}_{j} \partial \zeta}+\frac{1-\beta}{\zeta} \frac{\partial^{2} u}{\partial \zeta \partial \bar{z}_{j}}\right) \\
& -|\zeta|^{2-2 \beta} \sum_{j=1}^{n-1} \Gamma_{\zeta \zeta}^{l} \frac{\partial^{2} u}{\partial z_{l} \partial \bar{z}_{j}} \\
& -|\zeta|^{2-2 \beta}\left(\Gamma_{\zeta \zeta}^{\zeta}+\frac{1-\beta}{\zeta}\right) \frac{\partial^{2} u}{\partial \zeta \partial \bar{z}_{j}} .
\end{aligned}
$$

Since

$$
|\zeta|^{2-2 \beta}\left(\frac{\partial^{3} u}{\partial \zeta \partial \bar{z}_{j} \partial \zeta}+\frac{1-\beta}{\zeta} \frac{\partial^{2} u}{\partial \zeta \partial \bar{z}_{j}}\right)=O\left(|\zeta|^{\alpha \beta}\right)
$$

and

$$
|\zeta|^{1-\beta}\left(\Gamma_{\zeta \zeta}^{\zeta}+\frac{1-\beta}{\zeta}\right)=O\left(|\zeta|^{\alpha \beta}\right)
$$

we conclude that

$$
|\zeta|^{2-2 \beta}\left(D^{3} u\right)\left(\frac{\partial}{\partial \zeta}, \frac{\partial}{\partial \bar{z}_{j}}, \frac{\partial}{\partial \zeta}\right)=O\left(|\zeta|^{\alpha \beta}\right),
$$

as claimed. 
Proposition 6.5. We have

$$
|\zeta|^{3-3 \beta}\left(D^{3} u\right)\left(\frac{\partial}{\partial \zeta}, \frac{\partial}{\partial \bar{\zeta}}, \frac{\partial}{\partial \zeta}\right)=O\left(|\zeta|^{\alpha \beta}\right) .
$$

Proof. Differentiating the Monge-Ampère equation $\left(\star_{t}\right)$ gives

$$
\sum_{\mu, \nu=1}^{n} \hat{g}^{\mu \bar{\nu}}\left(D^{3} u\right)\left(\frac{\partial}{\partial \zeta}, \frac{\partial}{\partial \bar{z}_{\nu}}, \frac{\partial}{\partial z_{\mu}}\right)=\frac{\partial}{\partial \zeta}(t F) .
$$

Since the metric $\hat{\omega}$ is of class $\mathcal{C}^{, \alpha, \beta}$, we have

$$
\hat{g}^{k \bar{l}} \in \mathcal{C}^{, \alpha, \beta}, \quad|\zeta|^{\beta-1} \hat{g}^{k \bar{\zeta}} \in \mathcal{C}_{0}^{, \alpha, \beta}, \quad|\zeta|^{2 \beta-2} \hat{g}^{\zeta \bar{\zeta}} \in \mathcal{C}^{, \alpha, \beta} .
$$

Using Proposition 6.4, we obtain

$$
\begin{aligned}
& |\zeta|^{1-\beta} \hat{g}^{k \bar{l}}\left(D^{3} u\right)\left(\frac{\partial}{\partial \zeta}, \frac{\partial}{\partial \bar{z}_{l}}, \frac{\partial}{\partial z_{k}}\right)=O\left(|\zeta|^{\alpha \beta}\right), \\
& |\zeta|^{1-\beta} \hat{g}^{\zeta \bar{\zeta}}\left(D^{3} u\right)\left(\frac{\partial}{\partial \zeta}, \frac{\partial}{\partial \bar{z}_{l}}, \frac{\partial}{\partial \zeta}\right)=O\left(|\zeta|^{\alpha \beta}\right), \\
& |\zeta|^{1-\beta} \hat{g}^{k \bar{\zeta}}\left(D^{3} u\right)\left(\frac{\partial}{\partial \zeta}, \frac{\partial}{\partial \bar{\zeta}}, \frac{\partial}{\partial z_{k}}\right)=O\left(|\zeta|^{\alpha \beta}\right)
\end{aligned}
$$

for $k, l \in\{1, \ldots, n-1\}$. Substituting this into (13), we obtain

$$
|\zeta|^{1-\beta} \hat{g}^{\zeta \bar{\zeta}}\left(D^{3} u\right)\left(\frac{\partial}{\partial \zeta}, \frac{\partial}{\partial \bar{\zeta}}, \frac{\partial}{\partial \zeta}\right)=|\zeta|^{1-\beta} \frac{\partial}{\partial \zeta}(t F)+O\left(|\zeta|^{\alpha \beta}\right) .
$$

It follows from (8) that $|\zeta|^{1-\beta} \frac{\partial}{\partial \zeta} F=O\left(|\zeta|^{\beta}\right)$. Putting these facts together, we conclude that

$$
|\zeta|^{1-\beta} \hat{g}^{\zeta \bar{\zeta}}\left(D^{3} u\right)\left(\frac{\partial}{\partial \zeta}, \frac{\partial}{\partial \bar{\zeta}}, \frac{\partial}{\partial \zeta}\right)=O\left(|\zeta|^{\alpha \beta}\right) .
$$

This completes the proof.

After these preparations, we now describe the estimate for the covariant derivative of $\partial \bar{\partial} u$. Following Yau [15], we consider the function

$$
S=\sum_{\alpha, \beta, \gamma, \delta, \mu, \nu=1}^{n} \hat{g}^{\alpha \bar{\beta}} \hat{g}^{\gamma \bar{\delta}} \hat{g}^{\mu \bar{\nu}}\left(D^{3} u\right)\left(\frac{\partial}{\partial z_{\alpha}}, \frac{\partial}{\partial \bar{z}_{\delta}}, \frac{\partial}{\partial z_{\mu}}\right)\left(D^{3} u\right)\left(\frac{\partial}{\partial \bar{z}_{\beta}}, \frac{\partial}{\partial z_{\gamma}}, \frac{\partial}{\partial \bar{z}_{\nu}}\right) .
$$

Here, the tensor $D^{3} u$ represents the covariant derivative, with respect to $\omega$, of the (1,1)-form $\partial \bar{\partial} u$.

Proposition 6.6. Fix a point $p \in \Sigma$, and let $\left(z_{1}, \ldots, z_{n-1}, \zeta\right)$ be complex coordinates around $p$. Then

$$
S\left(z_{1}, \ldots, z_{n-1}, \zeta\right)=A\left(z_{1}, \ldots, z_{n-1}\right)+O\left(|\zeta|^{\alpha \beta}\right)
$$

where $A\left(z_{1}, \ldots, z_{n-1}\right)$ is a continuous function on $\Sigma$. 
Proof. It follows from Proposition 6.4 and Proposition 6.5 that the function $S$ can be written as a sum $S=S^{(1)}+S^{(2)}$, where $S^{(1)} \in \mathcal{C}^{, \alpha, \beta}$ and $S^{(2)}=O\left(|\zeta|^{\alpha \beta}\right)$. From this, the assertion follows.

Proposition 6.7. Let $u \in \mathcal{C}^{2, \alpha, \beta}$ be a solution of $\left(\star_{t}\right)$ for some $t \in[0,1]$. Then $S \leq C$ for some uniform constant $C$.

Proof. Let us fix $\varepsilon>0$ sufficiently small. It follows from Lemma 5.1 that

$$
\sqrt{-1} \partial \bar{\partial}\left(|s|_{h}^{2 \varepsilon}\right)+\omega \geq \varepsilon^{2}|s|_{h}^{2 \varepsilon} \sqrt{-1} \partial \log \left(|s|_{h}^{2}\right) \wedge \bar{\partial} \log \left(|s|_{h}^{2}\right)
$$

hence

$$
\Delta_{\hat{\omega}}\left(|s|_{h}^{2 \varepsilon}\right)+\operatorname{tr}_{\hat{\omega}}(\omega) \geq \varepsilon^{2}|s|_{h}^{2 \varepsilon}\left|d \log \left(|s|_{h}^{2}\right)\right|_{\hat{g}}^{2} .
$$

We have shown in Section 5 that $a_{1} \omega \leq \hat{\omega} \leq a_{2} \omega$ for uniform constants $a_{1}, a_{2}>0$. Thus, we conclude that

$$
\Delta_{\hat{\omega}}\left(|s|_{h}^{2 \varepsilon}\right) \geq \delta|s|_{h}^{2 \varepsilon-2 \beta}-K
$$

for uniform constants $\delta$ and $K$. We next consider the function $S$ defined above. Using the formula on page 266 of [2], we obtain

$$
\begin{aligned}
\Delta_{\hat{\omega}} S & \geq-C|D \partial \bar{\partial} u|_{g}^{2}|\partial \bar{\partial} F|_{g}-C|D \partial \bar{\partial} u|_{g}|D \partial \bar{\partial} F|_{g} \\
& -C|D \partial \bar{\partial} u|_{g}^{2}|R|_{g}-C|D \partial \bar{\partial} u|_{g}|D R|_{g},
\end{aligned}
$$

where $D$ denotes the Levi-Civita connection associated with $\omega$ (see also [15], Appendix A). Using the identity $\operatorname{Ric}_{\omega}=\sqrt{-1} \partial \bar{\partial} F$, we obtain

$$
\Delta_{\hat{\omega}} S \geq-C|D \partial \bar{\partial} u|_{g}^{2}|R|_{g}-C|D \partial \bar{\partial} u|_{g}|D R|_{g},
$$

hence

$$
\Delta_{\hat{\omega}} S \geq-C S|R|_{g}-C S^{\frac{1}{2}}|D R|_{g}
$$

Moreover, it follows from Proposition 3.4 and Proposition 3.5 that $|R|_{g} \leq C$ and $|D R|_{g} \leq C|s|_{h}^{\varepsilon-\beta}$. Putting these facts together, we obtain

$$
\Delta_{\hat{\omega}} S \geq-C|s|_{h}^{\varepsilon-\beta} S^{\frac{1}{2}}-C S .
$$

Therefore, we can find a uniform constant $L$ such that

$$
\Delta_{\hat{\omega}} S \geq-\delta|s|_{h}^{2 \varepsilon-2 \beta}-L S,
$$

where $\delta$ is the constant appearing in (15). Furthermore, equation (2.7) in [15] implies

$$
\Delta_{\hat{\omega}}\left(\Delta_{\omega} u\right) \geq \kappa S-N
$$

for uniform constants $\kappa$ and $N$. We next consider the function

$$
Q=S+\frac{L+1}{\kappa} \Delta_{\omega} u+|s|_{h}^{2 \varepsilon} .
$$

Using the inequalities (15), (16), and (17), we obtain

$$
\Delta_{\hat{\omega}} Q \geq S-\frac{L+1}{\kappa} N-K .
$$


Since $\Delta_{\omega} u$ is uniformly bounded, we conclude that

$$
\Delta_{\hat{\omega}} Q \geq Q-C
$$

for some uniform constant $C$. It follows from Proposition 6.6 that the function $Q$ attains its maximum at some point in $M \backslash \Sigma$. Using (18), we conclude that $Q$ is bounded from above by some uniform constant. From this, the assertion follows.

Corollary 6.8. Fix a point $p \in \Sigma$, and let $\left(z_{1}, \ldots, z_{n-1}, \zeta\right)$ be complex coordinates around $p$. Then the functions

$$
\frac{\partial^{2} u}{\partial z_{k} \partial \bar{z}_{l}}, \quad|\zeta|^{1-\beta} \frac{\partial^{2} u}{\partial \zeta \partial \bar{z}_{l}}, \quad|\zeta|^{2-2 \beta} \frac{\partial^{2} u}{\partial \zeta \partial \bar{\zeta}}
$$

are Lipschitz continuous with respect to $\omega$, and the Lipschitz constants are bounded from above by some uniform constant $L$. In particular, $\|u\|_{\mathcal{C}^{2, \alpha, \beta}} \leq$ $C$.

Proof. For abbreviation, let

$$
\varphi_{k l}=\frac{\partial^{2} u}{\partial z_{k} \partial \bar{z}_{l}}, \quad \psi_{l}=|\zeta|^{1-\beta} \frac{\partial^{2} u}{\partial \zeta \partial \bar{z}_{l}}, \quad \chi=|\zeta|^{2-2 \beta} \frac{\partial^{2} u}{\partial \zeta \partial \bar{\zeta}} .
$$

It follows from Proposition 6.7 that the covariant derivative of $\partial \bar{\partial} u$ is uniformly bounded, i.e. $|D \partial \bar{\partial} u|_{g} \leq C$ for some constant $C$. Moreover, we have

$$
\left|D \frac{\partial}{\partial z_{k}}\right|_{g} \leq C, \quad\left|D\left(\zeta^{1-\beta} \frac{\partial}{\partial \zeta}\right)\right|_{g} \leq C
$$

by Lemma 6.3. Here, $D$ denotes the Levi-Civita connection associated with the background edge metric $\omega$. Note that the vector field $\zeta^{1-\beta} \frac{\partial}{\partial \zeta}$ is defined only locally. Since

$$
\varphi_{k l}=\partial \bar{\partial} u\left(\frac{\partial}{\partial z_{k}}, \frac{\partial}{\partial \bar{z}_{l}}\right)
$$

we obtain

$$
\left|d \varphi_{k l}\right|_{g} \leq C
$$

Moreover, since

$$
\chi=\partial \bar{\partial} u\left(\zeta^{1-\beta} \frac{\partial}{\partial \zeta}, \overline{\zeta^{1-\beta} \frac{\partial}{\partial \zeta}}\right),
$$

we obtain

$$
|d \chi|_{g} \leq C .
$$

Finally, using the identity

$$
\frac{\zeta^{1-\beta}}{|\zeta|^{1-\beta}} \psi_{l}=\partial \bar{\partial} u\left(\zeta^{1-\beta} \frac{\partial}{\partial \zeta}, \frac{\partial}{\partial \bar{z}_{l}}\right),
$$


we obtain

$$
\left|d\left(\frac{\zeta^{1-\beta}}{|\zeta|^{1-\beta}} \psi_{l}\right)\right|_{g} \leq C .
$$

It follows from (19) and (20) that the functions $\varphi_{k l}$ and $\chi$ are Lipschitz continuous, with Lipschitz constant at most $C$. It remains to bound the Lipschitz constant of $\psi_{l}$. Note that the function $\psi_{l}$ vanishes along $\Sigma$. Integrating the inequality (21) along radial line segments yields $\left|\psi_{l}\left(z_{1}, \ldots, z_{n-1}, \zeta\right)\right| \leq$ $C|\zeta|^{\beta}$. Using this inequality and (21), we obtain

$$
\begin{aligned}
\left|d \psi_{l}\right|_{g} & \leq\left|d\left(\frac{\zeta^{1-\beta}}{|\zeta|^{1-\beta}} \psi_{l}\right)\right|_{g}+\left|\psi_{l}\right|\left|d\left(\frac{\zeta^{1-\beta}}{|\zeta|^{1-\beta}}\right)\right|_{g} \\
& \leq C+C|\zeta|^{\beta}\left|d\left(\frac{\zeta^{1-\beta}}{|\zeta|^{1-\beta}}\right)\right|_{g} \\
& \leq C+C|\zeta|^{\beta-1} \sqrt{g^{\zeta \bar{\zeta}}}
\end{aligned}
$$

Consequently, $\left|d \psi_{l}\right|_{g} \leq C$ for some uniform constant $C$. This completes the proof.

\section{Proof of Theorem 1.1}

We now finish the proof of Theorem 1.1. As above, we consider the equation

$$
(\omega+\sqrt{-1} \partial \bar{\partial} u)^{n}=e^{t F-c} \omega^{n},
$$

where $c$ is a constant. Moreover, let

$$
I=\left\{t \in[0,1]:\left(\star_{t}\right) \text { has a solution }(u, c) \in \mathcal{C}^{2, \alpha, \beta} \times \mathbb{R}\right\} .
$$

The equation $\left(\star_{0}\right)$ has the trivial solution $u=0$; in particular, $I$ is nonempty. It follows from Corollary 6.8 that any solution of $\left(\star_{t}\right)$ is uniformly bounded in $\mathcal{C}^{2, \alpha, \beta}$. Consequently, $I$ is closed. We next show that $I$ is open.

Proposition 7.1. The set $I$ is an open subset of $[0,1]$.

Proof. Fix a point $t_{0} \in I$. Moreover, let $u$ be a solution of $\left(\star_{t_{0}}\right)$, and let $\hat{\omega}=\omega+\sqrt{-1} \partial \bar{\partial} u$. By Theorem 3.7, the operator $\Delta_{\hat{\omega}}: \mathcal{C}^{2, \alpha, \beta} \rightarrow \mathcal{C}^{, \alpha, \beta}$ is Fredholm with Fredholm index zero. Consequently, the operator $\Delta_{\hat{\omega}}$ has one-dimensional cokernel and its range is given by $\left\{f \in \mathcal{C}^{, \alpha, \beta}: \int_{M} f \hat{\omega}^{n}=0\right\}$. Hence, the assertion follows from the implicit function theorem.

Corollary 7.2. We have $I=[0,1]$. In particular, the equation $\left(\star_{1}\right)$ admits a solution $u \in \mathcal{C}^{2, \alpha, \beta}$.

In the remainder of this section, we assume that $u \in \mathcal{C}^{2, \alpha, \beta}$ is a solution of $\left(\star_{1}\right)$. Then the associated Kähler metric $\hat{\omega}=\omega+\sqrt{-1} \partial \bar{\partial} u$ is Ricci flat. It remains to show that $\hat{\omega}$ has bounded curvature. The proof of this statement can be broken down into several lemmata. 
Lemma 7.3. Let $u \in \mathcal{C}^{2, \alpha, \beta}$ be a solution of $\left(\star_{1}\right)$. Then we have

$$
\begin{aligned}
& \frac{\partial^{2}}{\partial z_{i} \partial \bar{z}_{j}} \hat{g}_{k \bar{l}} \in \mathcal{C}^{, \alpha, \beta} \\
& |\zeta|^{1-\beta} \frac{\partial}{\partial z_{i} \partial \bar{\zeta}} \hat{g}_{k \bar{l}} \in \mathcal{C}_{0}^{, \alpha, \beta} \\
& |\zeta|^{1-\beta} \frac{\partial}{\partial \zeta \partial \bar{z}_{j}} \hat{g}_{k \bar{l}} \in \mathcal{C}_{0}^{, \alpha, \beta} \\
& |\zeta|^{2-2 \beta} \frac{\partial}{\partial \zeta \partial \bar{g}_{k \bar{l}} \in \hat{\mathcal{C}}^{, \alpha, \beta}}
\end{aligned}
$$

for all $i, j, k, l \in\{1, \ldots, n-1\}$.

Proof. Let us fix two integers $k, l \in\{1, \ldots, n-1\}$. Since $u$ is a solution of $\left(\star_{1}\right)$, the Ricci tensor of $\hat{g}$ vanishes. This implies

$$
\Delta_{\hat{\omega}} \hat{g}_{k \bar{l}}-\sum_{\alpha, \beta, \mu, \nu=1}^{n} \hat{g}^{\alpha \bar{\beta}} \hat{g}^{\mu \bar{\nu}} \frac{\partial}{\partial z_{k}} \hat{g}_{\alpha \bar{\nu}} \frac{\partial}{\partial \bar{z} l} \hat{g}_{\mu \bar{\beta}}=0 .
$$

By Lemma 6.1, the $(1,1)$-forms $\frac{\partial}{\partial z_{k}} \hat{\omega}$ and $\frac{\partial}{\partial \bar{z}_{l}} \hat{\omega}$ are of class $\mathcal{C}^{, \alpha, \beta}$. Consequently, we can find a function $f \in \mathcal{C}, \alpha, \beta$ such that $\Delta_{\hat{\omega}} \hat{g}_{k \bar{l}}=f$ away from $\Sigma$. Hence, the assertion follows from Corollary 2.6.

Lemma 7.4. Let $u \in \mathcal{C}^{2, \alpha, \beta}$ be a solution of $\left(\star_{1}\right)$. Then we have

$$
|\zeta|^{2-2 \beta}\left(\frac{\partial^{4} u}{\partial \zeta \partial \bar{z}_{j} \partial \zeta \partial \bar{z}_{l}}+\frac{1-\beta}{\zeta} \frac{\partial^{3} u}{\partial \bar{z}_{j} \partial \zeta \partial \bar{z}_{l}}\right)=O\left(|\zeta|^{\alpha \beta}\right)
$$

for all $j, l \in\{1, \ldots, n-1\}$.

Proof. Fix two integers $j, l \in\{1, \ldots, n-1\}$. By assumption, we have $u \in \mathcal{C}^{2, \alpha, \beta}$. In particular, we have $\Delta_{\Omega} u \in \mathcal{C}^{, \alpha, \beta}$. Using the results in [3], we conclude that $\frac{\partial^{2} u}{\partial \bar{z}_{j} \partial \bar{z}_{l}} \in \mathcal{C}^{, \alpha, \beta}$. Since $u$ is a solution of $\left(\star_{1}\right)$, we have

$$
\frac{\partial^{2}}{\partial \bar{z}_{j} \partial \bar{z}_{l}} \log \hat{\omega}^{n}=\frac{\partial^{2}}{\partial \bar{z}_{j} \partial \bar{z}_{l}}\left(\log \omega^{n}+F\right)
$$

This implies

$$
\begin{aligned}
& \Delta_{\hat{\omega}}\left(\frac{\partial^{2} u}{\partial \bar{z}_{j} \partial \bar{z}_{l}}\right)+\sum_{\mu, \nu=1}^{n} \hat{g}^{\mu \bar{\nu}} \frac{\partial^{2}}{\partial \bar{z}_{j} \partial \bar{z}_{l}} g_{\mu \bar{\nu}}-\sum_{\alpha, \beta, \mu, \nu=1}^{n} \hat{g}^{\alpha \bar{\beta}} \hat{g}^{\mu \bar{\nu}} \frac{\partial}{\partial \bar{z}_{j}} \hat{g}_{\alpha \bar{\nu}} \frac{\partial}{\partial \bar{z}_{l}} \hat{g}_{\mu \bar{\beta}} \\
& =\sum_{\mu, \nu=1}^{n} \hat{g}^{\mu \bar{\nu}} \frac{\partial^{2}}{\partial \bar{z}_{j} \partial \bar{z}_{l}} \hat{g}_{\mu \bar{\nu}}-\sum_{\alpha, \beta, \mu, \nu=1}^{n} \hat{g}^{\alpha \bar{\beta}} \hat{g}^{\mu \bar{\nu}} \frac{\partial}{\partial \bar{z}_{j}} \hat{g}_{\alpha \bar{\nu}} \frac{\partial}{\partial \bar{z}_{l}} \hat{g}_{\mu \bar{\beta}} \\
& =\frac{\partial^{2}}{\partial \bar{z}_{j} \partial \bar{z}_{l}}\left(\log \omega^{n}+F\right) .
\end{aligned}
$$


By Lemma 6.1, the $(1,1)$-forms $\frac{\partial}{\partial \bar{z}_{j}} \hat{\omega}$ and $\frac{\partial}{\partial \bar{z}_{l}} \hat{\omega}$ are of class $\mathcal{C}, \alpha, \beta$. Moreover, it is easy to see that the function $\frac{\partial^{2}}{\partial \bar{z}_{j} \partial \bar{z}_{l}}\left(\log \omega^{n}+F\right)$ is of class $\mathcal{C}^{, \alpha, \beta}$. Consequently, there exists a function $f \in \mathcal{C}^{, \alpha, \beta}$ such that $\Delta_{\hat{\omega}}\left(\frac{\partial^{2} u}{\partial \bar{z}_{j} \partial \bar{z}_{l}}\right)=f$ away from $\Sigma$. Using Corollary 2.6, we conclude that

$$
\frac{\partial^{2} u}{\partial \bar{z}_{j} \partial \bar{z}_{l}} \in \mathcal{C}^{2, \alpha, \beta}
$$

In particular, we have

$$
|\zeta|^{2-2 \beta} \frac{\partial^{4} u}{\partial \zeta \partial \bar{\zeta} \partial \bar{z}_{j} \partial \bar{z}_{l}} \in \mathcal{C}^{, \alpha, \beta}
$$

Hence, the assertion follows from Proposition A.1.

Proposition 7.5. Let $u \in \mathcal{C}^{2, \alpha, \beta}$ be a solution of $\left(\star_{1}\right)$, and let $\hat{R}$ denote the Riemann curvature tensor of $\hat{g}$. Then $\sup _{M \backslash \Sigma}|\hat{R}|_{\hat{g}}<\infty$.

Proof. As in Section 3, we consider the map

$$
\Psi:\left(z_{1}, \ldots, z_{n-1}, z_{n}\right) \mapsto\left(z_{1}, \ldots, z_{n-1}, z_{n}^{\frac{1}{\beta}}\right) .
$$

We have shown in Section [6 that $|D \hat{\omega}|_{g}=|D \partial \bar{\partial} u|_{g}=O(1)$. This implies

$$
\sum_{\alpha, \gamma, \delta=1}^{n}\left|\frac{\partial}{\partial z_{\alpha}} \Psi^{*} \hat{g}\left(\frac{\partial}{\partial z_{\gamma}}, \frac{\partial}{\partial \bar{z}_{\delta}}\right)\right|=O(1) .
$$

Using Lemma 7.3, we obtain

$$
\sum_{i, j, k, l=1}^{n-1}\left|\frac{\partial^{2}}{\partial z_{i} \partial \bar{z}_{j}} \Psi^{*} \hat{g}\left(\frac{\partial}{\partial z_{k}}, \frac{\partial}{\partial \bar{z}_{l}}\right)\right|=O(1)
$$

and

$$
\sum_{j, k, l=1}^{n-1}\left|\frac{\partial^{2}}{\partial z_{n} \partial \bar{z}_{j}} \Psi^{*} \hat{g}\left(\frac{\partial}{\partial z_{k}}, \frac{\partial}{\partial \bar{z}_{l}}\right)\right|=O(1) .
$$

Consequently, the Riemann curvature tensor of $\hat{g}$ satisfies

$$
\sum_{i, j, k, l=1}^{n-1}\left|\hat{R}\left(\frac{\partial}{\partial z_{i}}, \frac{\partial}{\partial \bar{z}_{j}}, \frac{\partial}{\partial z_{k}}, \frac{\partial}{\partial \bar{z}_{l}}\right)\right|=O(1)
$$

and

$$
\sum_{j, k, l=1}^{n-1}\left|\hat{R}\left(\frac{\partial}{\partial z_{n}}, \frac{\partial}{\partial \bar{z}_{j}}, \frac{\partial}{\partial z_{k}}, \frac{\partial}{\partial \bar{z}_{l}}\right)\right|=O(1) .
$$

Moreover, Lemma 7.4 implies that

$$
\sum_{j, l=1}^{n-1}\left|\frac{\partial^{4}}{\partial z_{n} \partial \bar{z}_{j} \partial z_{n} \partial \bar{z}_{l}}(u \circ \Psi)\right|=O(1) .
$$


Using this inequality and the estimate

$$
\sum_{j, l=1}^{n-1}\left|\frac{\partial^{2}}{\partial z_{n} \partial \bar{z}_{j}} \Psi^{*} g\left(\frac{\partial}{\partial z_{n}}, \frac{\partial}{\partial \bar{z}_{l}}\right)\right|=O(1),
$$

we obtain

$$
\sum_{j, l=1}^{n-1}\left|\frac{\partial^{2}}{\partial z_{n} \partial \bar{z}_{j}} \Psi^{*} \hat{g}\left(\frac{\partial}{\partial z_{n}}, \frac{\partial}{\partial \bar{z}_{l}}\right)\right|=O(1) .
$$

Thus, we conclude that

$$
\sum_{j, l=1}^{n-1}\left|\hat{R}\left(\frac{\partial}{\partial z_{n}}, \frac{\partial}{\partial \bar{z}_{j}}, \frac{\partial}{\partial z_{n}}, \frac{\partial}{\partial \bar{z}_{l}}\right)\right|=O(1) .
$$

Since the Ricci tensor of $\hat{g}$ vanishes, the inequalities (22), (223), and (24) imply that

$$
\sum_{\alpha, \beta, \gamma, \delta=1}^{n}\left|\hat{R}\left(\frac{\partial}{\partial z_{\alpha}}, \frac{\partial}{\partial \bar{z}_{\beta}}, \frac{\partial}{\partial z_{\gamma}}, \frac{\partial}{\partial \bar{z}_{\delta}}\right)\right|=O(1) .
$$

Since the metric $\hat{g}$ is uniformly equivalent to $g$, we conclude that $|\hat{R}|_{\hat{g}}=O(1)$. This completes the proof of Proposition 7.5.

\section{Appendix A. An Auxiliary Result}

Proposition A.1. Fix real numbers $\alpha \in(0,1)$ and $\beta \in\left(0, \frac{1}{2}\right)$ such that $\alpha \beta<1-2 \beta$. Assume that $\tilde{f}$ is a function defined on the unit disk $B_{1}(0) \subset \mathbb{C}$ of class $C^{\alpha}$. Moreover, suppose that $v$ is a smooth function defined on the punctured disk $B_{1}(0) \backslash\{0\} \subset \mathbb{C}$ satisfying $\sup _{B_{1}(0) \backslash\{0\}}|v|<\infty$ and

$$
|\zeta|^{2-2 \beta} \frac{\partial^{2} v}{\partial \zeta \partial \bar{\zeta}}=\tilde{f}\left(|\zeta|^{\beta-1} \zeta\right)
$$

on $B_{1}(0) \backslash\{0\}$. Then

$$
|\zeta|^{2-2 \beta}\left(\frac{\partial^{2} v}{\partial \zeta^{2}}+\frac{1-\beta}{\zeta} \frac{\partial v}{\partial \zeta}\right)=O\left(|\zeta|^{\alpha \beta}\right)
$$

Proof. Let

$$
w(\zeta)=v(\zeta)-\frac{\tilde{f}(0)}{\beta^{2}}|\zeta|^{2 \beta}
$$

and

$$
h(\zeta)=|\zeta|^{2 \beta-2}\left(\tilde{f}\left(|\zeta|^{\beta-1} \zeta\right)-\tilde{f}(0)\right)
$$

Since $\tilde{f}$ is of class $C^{\alpha}$, we have $|h(\zeta)| \leq C|\zeta|^{2 \beta-2+\alpha \beta}$. Moreover, we have

$$
\frac{\partial^{2} w}{\partial \zeta \partial \bar{\zeta}}=h
$$


on $B_{1}(0) \backslash\{0\}$. Since $\sup _{B_{1}(0) \backslash\{0\}}|w|<\infty$, the previous equation holds in the distributional sense. Using Green's formula, we obtain

$$
\begin{aligned}
\left|\frac{\partial w}{\partial \zeta}\left(\zeta_{0}\right)\right| & \leq C \int_{B_{\frac{1}{2}}(0)}\left|\zeta-\zeta_{0}\right|^{-1}|h(\zeta)| d \zeta+C \\
& \leq C \int_{B_{\frac{1}{2}}(0)}\left|\zeta-\zeta_{0}\right|^{-1}|\zeta|^{2 \beta-2+\alpha \beta} d \zeta+C \\
& \leq C\left|\zeta_{0}\right|^{2 \beta-1+\alpha \beta} .
\end{aligned}
$$

for $\zeta_{0} \in B_{1 / 4}(0)$. Consequently,

$$
|\zeta|^{1-2 \beta} \frac{\partial w}{\partial \zeta}=O\left(|\zeta|^{\alpha \beta}\right)
$$

We now consider a point $\zeta_{0} \in B_{1 / 4}(0)$. Since $\tilde{f}$ is of class $C^{\alpha}$, we have

$$
\begin{aligned}
\left|h\left(\zeta_{1}\right)-h\left(\zeta_{2}\right)\right| & \leq\left|\zeta_{1}\right|^{2 \beta-2}\left|\tilde{f}\left(\left|\zeta_{1}\right|^{\beta-1} \zeta_{1}\right)-\tilde{f}\left(\left|\zeta_{2}\right|^{\beta-1} \zeta_{2}\right)\right| \\
& +\left.|| \zeta_{1}\right|^{2 \beta-2}-\left|\zeta_{2}\right|^{2 \beta-2}|| \tilde{f}\left(\left|\zeta_{2}\right|^{\beta-1} \zeta_{2}\right)-\tilde{f}(0) \mid \\
& \leq\left. C\left|\zeta_{1}\right|^{2 \beta-2}|| \zeta_{1}\right|^{\beta-1} \zeta_{1}-\left.\left|\zeta_{2}\right|^{\beta-1} \zeta_{2}\right|^{\alpha} \\
& +\left.C|| \zeta_{1}\right|^{2 \beta-2}-\left.\left|\zeta_{2}\right|^{2 \beta-2}|| \zeta_{2}\right|^{\alpha \beta}
\end{aligned}
$$

for all $\zeta_{1}, \zeta_{2} \in B_{\left|\zeta_{0}\right| / 2}\left(\zeta_{0}\right)$. This implies

$$
\begin{aligned}
\left|h\left(\zeta_{1}\right)-h\left(\zeta_{2}\right)\right| & \leq C\left|\zeta_{0}\right|^{2 \beta-2+\alpha(\beta-1)}\left|\zeta_{1}-\zeta_{2}\right|^{\alpha} \\
& +C\left|\zeta_{0}\right|^{2 \beta-3+\alpha \beta}\left|\zeta_{1}-\zeta_{2}\right|
\end{aligned}
$$

for all $\zeta_{1}, \zeta_{2} \in B_{\left|\zeta_{0}\right| / 2}\left(\zeta_{0}\right)$. Therefore,

$$
[h]_{C^{\alpha}\left(B_{\left|\zeta_{0}\right| / 2}\left(\zeta_{0}\right)\right)} \leq C\left|\zeta_{0}\right|^{2 \beta-2+\alpha(\beta-1)} .
$$

Using standard interior estimates, we obtain

$$
\begin{aligned}
\left|\frac{\partial^{2} w}{\partial \zeta^{2}}\left(\zeta_{0}\right)\right| & \leq C\left|\zeta_{0}\right|^{-1} \sup _{B_{\left|\zeta_{0}\right| / 2}\left(\zeta_{0}\right)}\left|\frac{\partial w}{\partial \zeta}\right| \\
& +C \sup _{B_{\left|\zeta_{0}\right| / 2}\left(\zeta_{0}\right)}|h|+C\left|\zeta_{0}\right|^{\alpha}[h]_{C^{\alpha}\left(B_{\left|\zeta_{0}\right| / 2}\left(\zeta_{0}\right)\right)} .
\end{aligned}
$$

Putting these facts together, we conclude that

$$
\left|\frac{\partial^{2} w}{\partial \zeta^{2}}\left(\zeta_{0}\right)\right| \leq C\left|\zeta_{0}\right|^{2 \beta-2+\alpha \beta}
$$

Consequently,

$$
|\zeta|^{2-2 \beta} \frac{\partial^{2} w}{\partial \zeta^{2}}=O\left(|\zeta|^{\alpha \beta}\right)
$$

Putting these facts together, we obtain

$$
|\zeta|^{2-2 \beta}\left(\frac{\partial^{2} v}{\partial \zeta^{2}}+\frac{1-\beta}{\zeta} \frac{\partial v}{\partial \zeta}\right)=|\zeta|^{2-2 \beta}\left(\frac{\partial^{2} w}{\partial \zeta^{2}}+\frac{1-\beta}{\zeta} \frac{\partial w}{\partial \zeta}\right)=O\left(|\zeta|^{\alpha \beta}\right) .
$$




\section{REFERENCES}

[1] T. Aubin, Équations du type Monge-Ampère sur les variétés Kähleriennes compactes, C.R. Acad. Sci. Paris Sér. A-B 283, A119-A121 (1976)

[2] T. Aubin, Some nonlinear problems in Riemannian geometry, Springer Monographs in Mathematics, Springer, 1998

[3] S. Donaldson, Kähler metrics with cone singularities along a divisor, arXiv:1102.1196

[4] D. Gilbarg and N. Trudinger, Elliptic Partial Differential Equations of Second Order, Springer-Verlag, 2001

[5] T. Jeffres, Schwarz lemma for Kähler cone metrics, Internat. Math. Res. Notices 371-382 (2000)

[6] T. Jeffres, Uniqueness of Kähler-Einstein cone metrics, Publ. Mat. 44, 437-448 (2000)

[7] R. Mazzeo, Elliptic theory of differential edge operators, I, Comm. Partial Differential Equations 16, 1615-1664 (1991)

[8] R. Mazzeo, Kähler-Einstein metrics singular along a smooth divisor, Journées Équations aux Dérivées Partielles (Saint-Jean-de-Monts, 1999), Exp. No. VI, 1-10

[9] L. Simon, Schauder estimates by scaling, Calc. Var. PDE 5, 391-407 (1997)

[10] G. Tian, Canonical metrics in Kähler geometry, Birkhäuser, 2000

[11] G. Tian and S.T. Yau, Complete Kähler manifolds with zero Ricci curvature I, J. Amer. Math. Soc. 3, 579-609 (1990)

[12] G. Tian and S.T. Yau, Complete Kähler manifolds with zero Ricci curvature II, Invent. Math. 106, 27-60 (1991)

[13] M. Troyanov, Prescribing curvature on compact surfaces with conical singularities, Trans. Amer. Math. Soc. 325, 793-821 (1991)

[14] S.T. Yau, On Calabi's conjecture and some new results in algebraic geometry, Proc. Natl. Acad. Sci. USA 74, 1798-1799 (1977)

[15] S.T. Yau, On the Ricci curvature of a compact Kähler manifold and the complex Monge-Ampère equation, Comm. Pure Appl. Math. 31, 339-411 (1978) 